\title{
Non-existence of patterns and gradient estimates in semilinear elliptic equations with Neumann boundary conditions
}

\author{
Samuel Nordmann*
}

September 29, 2020

\begin{abstract}
We call pattern any non-constant solution of a semilinear elliptic equation with Neumann boundary conditions. A classical theorem of Casten, Holland 20] and Matano [50] states that stable patterns do not exist in convex domains. In this article, we show that the assumptions of convexity of the domain and stability of the pattern in this theorem can be relaxed in several directions. In particular, we propose a general criterion for the non-existence of patterns, dealing with possibly non-convex domains and unstable patterns. Our results unfold the interplay between the geometry of the domain, the stability of patterns, and the $C^{1}$ norm of the nonlinearity.

In addition, we establish several gradient estimates for the patterns of (1). We prove a general nonlinear Cacciopoli inequality (or an inverse Poincaré inequality), stating that the $L^{2}$-norm of the gradient of a solution is controlled by the $L^{2}$-norm of $f(u)$, with a constant that only depends on the domain. This inequality holds for non-homogeneous equations. We also give several flatness estimates.

Our approach relies on the introduction of what we call the Robincurvature Laplacian. This operator is intrinsic to the domain and contains much information on how the geometry of the domain affects the shape of the solutions.

Finally, we extend our results to unbounded domains. It allows us to improve the results of our previous paper [54] and to extend some results on De Giorgi's conjecture to a larger class of domains.
\end{abstract}

Keywords: Stability ; Cacciopoli Inequality ; De Giorgi's conjecture ; Liouville type results; Robin-curvature Laplacian ; Flatness Estimate ; Spectral Gap ; Semilinear elliptic equations ; Symmetry ; Neumann boundary conditions ; Indefinite Robin Boundary Conditions

AMS Class. No: 35B35, 35B06, 35J15, 35J61, 35B53.

*samnordmann@gmail.com ; School of Mathematical Sciences, Tel Aviv University. 
Acknowledgement. The author is deeply thankful to Professor Henri Berestycki for many very instructive discussions.

The research leading to these results has received funding from the European Research Council under the European Union's Seventh Framework Programme (FP/2007-2013) / ERC Grant Agreement n.321186 - ReaDi -ReactionDiffusion Equations, Propagation and Modelling held by Henri Berestycki.

\section{Introduction}

We call pattern any non-constant solution $u \in C^{3}(\bar{\Omega})$ of the following equation

$$
\begin{cases}-\Delta u(x)=f(u(x)) & \forall x \in \Omega, \\ \partial_{\nu} u(x)=0 & \forall x \in \partial \Omega,\end{cases}
$$

where the domain $\Omega \subset \mathbb{R}^{n}(n \geq 2)$ is bounded and smooth (say, $C^{2}$ ), $\partial_{\nu}$ is the outward normal derivative, and the nonlinearity $f$ is $C^{1}$. We sometimes consider the non-homogeneous case, that is, we sometimes allow $f=f(x, u)$ to depend on $x \in \Omega$.

Note that if $z \in \mathbb{R}$ is a root of $f$, then $z$ is a constant solution of the above equation (but it is not a pattern).

We are interested in the existence/non-existence and gradient estimates of patterns. Our starting point is a celebrated theorem proved independently by Casten, Holland [20], and by Matano [50].

Theorem CHM ([20,50]). If the domain is convex, there exists no stable pattern to (1).

Here, a solution is said to be stable if the second variation of energy is nonnegative (see Definition 2 below).

Theorem CHM states that, if the domain is convex, any stable solution is constant. This result has many consequences on the classification of solutions, the asymptotics of the associated evolution problem, the construction of traveling fronts [13], and has also many applications in chemistry, population dynamics, etc. Theorem CHM has been extended in several directions: certain non-linear elliptic operators $[24,32$, Robin boundary conditions 6,32 , manifolds [7, 31, 45], unbounded convex domains [54] and some systems [46, 71]. See also 33. We give in Section 3 a more detailed presentation of the literature on this topic.

The present article aims to investigate whether the assumptions of convexity of the domain and stability of the solution can be relaxed in Theorem CHM. To our knowledge, the literature does not contain any result in this direction. In particular, no sufficient condition is known for the non-existence of patterns in non-convex domains.

Apparently, the assumptions of Theorem CHM are sharp in general. On the one hand, the conclusion of Theorem CHM does not hold for unstable patterns, since $u(x)=\cos (x)$ is an unstable pattern of (1) for $\Omega=x \in[0, \pi]$ and $f(u)=u$. 
On the other hand, we cannot relax the assumption of convexity of the domain, since a result of Kohn and Sternberg [47. allows constructing stable patterns in some star-shaped domains obtained as a small perturbation of a convex domain. These domains typically feature a bottleneck which is sufficiently narrow compared to $f$, see Section 3 for more details.

Nevertheless, when the domain is almost convex or the solution is almost stable, the construction of a stable pattern needs the nonlinearity to have large variations. This suggests that, in non-convex domains, the non-existence of patterns involves the geometry of the domain, the stability of the solution and the $C^{1}$ norm of the nonlinearity.

In this paper, we improve and extend Theorem CHM in several directions. First, we show that the two assumptions of convexity of the domain and stability of the patterns can be combined in a single assumption on the spectrum of a certain operator (Theorem 1). Then, we propose a general criterion for the non-existence of patterns (Theorem 2), which deals with possibly non-convex domains and possibly unstable patterns. To the best of our knowledge, this is the first result of this type. This criterion unfolds the interplay between the geometry of the domain, the stability of patterns and the $C^{1}$ norm of the nonlinearity. We propose several applications, in particular, we prove that Theorem CHM is robust under smooth perturbations of the domain and the nonlinearity (Proposition 1).

We establish in this paper several gradient estimates for the patterns of (1). We prove a general nonlinear Cacciopoli inequality (or an inverse Poincaré inequality), stating that the $L^{2}$-norm of the gradient of a solution is controlled by the $L^{2}$-norm of $f(u)$, with a constant that only depends on the domain (Theorem 3). This inequality holds for non-homogeneous equations (i.e., when $f=f(x, u)$ depends on $x \in \Omega)$. To the best of our knowledge, this is a new result. Also, we give several flatness estimates in term of the spectral gap of some geometric operator (Proposition 2) or the Morse index of the solution (Proposition 3).

Finally, we extend our main results to unbounded domains. We refine the results of our previous paper [54] and extend some results on De Giorgi's conjecture to a larger class of domains.

We point out that the conclusion of Theorem CHM holds for any $C^{1}$ nonlinearity. Throughout the paper, we shall stick to this general context by making no further assumption on the nonlinearity. However, let us mention that Theorem CHM (and also our results) can be improved if the nonlinearity satisfies certain additional properties:

- if $f$ is convex or concave, there exists no stable pattern in any (possibly non-convex) domain [20].

- if $f^{\prime} \leq 0$ (resp. $f^{\prime}>0$ ), any solution of (1) is stable (resp. unstable), see Definition 2 below. 
- if $f \geq 0$ or $f \leq 0$, there exists no (possibly unstable) patterns. To prove this, note that integrating equation (1) implies $\int_{\Omega} f=0$, and so $f(u(x))=$ 0 . Then, we conclude with the strong maximum principle.

- if $\frac{f(u)}{u^{p^{x}-1}}$ is non increasing, there exist no (possiby unstable) patterns in convex domains 24]. This is related to the Lin-Ni conjecture, see [68 and references therein.

In our context, bistable nonlinearities (e.g. $f(u)=-(u-a)(u-b)(u-c)$, with $a<b<c)$ are especially interesting since they favor the existence of stable patterns, especially the Allen-Cahn nonlinearity $f(u)=u-u^{3}$ which is balanced between its two stable roots (i.e. $\int_{-1}^{1} f=0$ ).

Our approach relies on the introduction and analysis of what we call the Robin-curvature Laplacian.

Definition 1 (Robin-curvature Laplacian). We denote by $\gamma(x)$ the lowest principal curvature of the domain at $x \in \partial \Omega$ (i.e., $\gamma(x)$ is the lowest eigenvalue of the second fundamental form). In dimension $n=2$, then $\gamma(\cdot)$ is nothing but the curvature of $\partial \Omega$.

We define the Robin-curvature Laplacian as the Laplace operator acting on $\psi \in C^{2}(\bar{\Omega})$ associated with the boundary conditions

$$
\partial_{\nu} \psi+\gamma \psi=0 .
$$

We also define its lowest eigenvalue

$$
\mu_{\gamma}:=\inf _{\substack{\psi \in H^{1} \\\|\psi\|_{L^{2}}=1}} \mathcal{G}_{\gamma}(\psi):=\inf _{\substack{\psi \in H^{1} \\\|\psi\|_{L^{2}}=1}} \int_{\Omega}|\nabla \psi|^{2}+\int_{\partial \Omega} \gamma \psi^{2},
$$

and more generally, for $a \geq 0$,

$$
\mu_{a \gamma}:=\inf _{\substack{\psi \in H^{1} \\\|\psi\|_{L^{2}}=1}} \mathcal{G}_{a \gamma}(\psi):=\inf _{\substack{\psi \in H^{1} \\\|\psi\|_{L^{2}}=1}} \int_{\Omega}|\nabla \psi|^{2}+a \int_{\partial \Omega} \gamma \psi^{2} .
$$

We emphasize that the Robin-curvature Laplacian is intrinsic to the domain and does not involve the nonlinearity. To the best of our knowledge, this operator has not been considered before in the literature. Note that $\gamma \geq 0$ if and only if $\Omega$ is convex. Thus, if $\Omega$ is convex, the Robin-curvature Laplacian involves classical Robin boundary conditions with a nonnegative $\gamma$. If $\Omega$ is not convex, then $\gamma$ changes sign and the Robin boundary conditions are indefinite. As a consequence of the work of Daners [30], the Robin-curvature Laplacian satisfies most of the classical properties known for the definite Robin Laplacian. In particular, its principal eigenvalue $\mu_{\gamma}$ is well defined and finite. Let us point out that the term $\int_{\partial \Omega} \gamma \psi^{2}$ could be interpreted as the surface tension of a body $\Omega$ with rigidity on its surface.

Our results reveal that the Robin-curvature Laplacian contains much information on how the geometry of the domain affects the shape of the solutions of (11). An in-depth study of this operator could lead to new results. 
Outline. The results are presented in Section 2 In Section 3, we discuss the existing literature and the implications of our results. The remaining of the paper is devoted to the proofs of our results. In Section 4, we prove the results dealing with the non-existence of patterns Theorem 1, Proposition 1 and Theorem 2). Section 5 is devoted to the proof of the nonlinear Cacciopoli inequality (Theorem 3). In Section 6, we prove the flatness estimates (Proposition 2 and Proposition 3. Finally, in Section 7 we extend our main results to unbounded domains (Theorem 4, Corollary 3 and Theorem 5).

\section{Presentation of the results}

\section{$2.1 \quad$ Non-existence of patterns}

The first step in our approach relies in the observation that the two assumptions of Theorem CHM (that is, the stability of the solution and the convexity of the domain) can be combined in a single assumption on what we call the Robincurvature-stability of the solution.

Before presenting our first result, let us define precisely the stability of a solution.

Definition 2 (Stability). A solution $u$ of (1) is said to be stable if the second variation of energy at $u$ is nonnegative, namely, if

$$
\lambda_{0}:=\inf _{\substack{\psi \in H^{1} \\\|\psi\|_{L^{2}}=1}} \mathcal{F}_{0}(\psi):=\inf _{\substack{\psi \in H^{1} \\\|\psi\|_{L^{2}}=1}} \int_{\Omega}|\nabla \psi|^{2}-\int_{\Omega} f^{\prime}(u) \psi^{2} \geq 0 .
$$

Note that $\lambda_{0}$ is the lowest eigenvalue of the linearized of operator of (1) at $u$. According to this definition, any local minimum or degenerate critical point of the energy is stable. Therefore, the set of stable solutions contains all the steady states obtained as a (strict) limit of an evolution problem.

Let us now define what we call the Robin-curvature-stability of a solution.

Definition 3 (Robin-curvature-stability). Let u be a solution of (1). Set

$$
\lambda_{\gamma}:=\inf _{\substack{\psi \in H^{1}(\Omega) \\\|\psi\|_{L^{2}}=1}} \mathcal{F}_{\gamma}(\psi):=\inf _{\substack{\psi \in H^{1}(\Omega) \\\|\psi\|_{L^{2}}=1}} \int_{\Omega}|\nabla \psi|^{2}-\int_{\Omega} f^{\prime}(u) \psi^{2}+\int_{\partial \Omega} \gamma \psi^{2},
$$

where $\gamma$ is the minimal curvature of the domain (Definition 1. In analogy with Definition 2, we say that $u$ is Robin-curvature-stable if $\lambda_{\gamma} \geq 0$.

We point out that $\lambda_{\gamma}$ is the principal eigenvalue of the linearized operator at $u$, for which we have replaced the Neumann boundary conditions with the Robin-curvature boundary conditions $\partial_{\nu} \psi+\gamma \psi=0$. Note that our notation is consistent with Definition 2 since $\lambda_{\gamma}$ coincides with $\lambda_{0}$ if we formally take $\gamma=0$ in the above definition. The definitions of $\lambda_{0}$ and $\lambda_{\gamma}$ differ by the addition of the term $\int_{\partial \Omega} \gamma \psi^{2}$. This justifies the terminology Robin-curvature stability. However, 
the Robin-curvature-stability do not refer to a usual notion of stability, because $u$ is not a critical point of the Robin-curvature energy $\mathcal{E}_{\gamma}(u)=\int_{\Omega} \frac{1}{2}|\nabla u|^{2}-$ $F(u)+\int_{\partial \Omega} \gamma u^{2}$ with $F^{\prime}=f$.

We are ready to state a key observation.

Theorem 1. There exists no Robin-curvature-stable patterns to (1).

We point out that this conclusion holds for possibly unstable patterns and non-convex domains.

Theorem CHM is a special case of the above theorem. Indeed, recalling that $\Omega$ is convex if, and only if, $\gamma \geq 0$, we see immediately that, for $u$ a solution of (1),

$$
\text { if } \Omega \text { is convex, then } \lambda_{\gamma} \geq \lambda_{0}
$$

(in fact, the strict inequality holds in (4), see Lemma 3); hence, in a convex domain, stability implies Robin-curvature-stability.

The Robin-curvature-stability $\left(\lambda_{\gamma} \geq 0\right)$ happens to combine the two assumptions of Theorem CHM namely, the stability $\left(\lambda_{0} \geq 0\right)$ and the convexity of the domain $(\gamma \geq 0)$. As a first consequence of Theorem 1 we establish the robustness of Theorem CHM under smooth perturbations of the domain and the nonlinearity.

Proposition 1. Let $\Omega$ be a bounded smooth convex domain and $f$ a $C^{1}$ nonlinearity. If $\left(\Omega_{\varepsilon}, f_{\varepsilon}\right)$ is a smooth perturbation of $(\Omega, f)$, there exists no stable pattern to (1).

The perturbation is understood to be such that $(\Omega, f) \mapsto \lambda_{\gamma}$ is continuous. It is classical [44] (see also $27,28,42]$ ) that this is the case under a $C^{1}$ perturbation on the nonlinearity and a small $C^{2, \alpha}$ modification of the boundary of the domain (whereas it may fail under rougher perturbations [29]).

As further explained in Section 3, a result of Kohn and Sternberg [47 implies that there exist patterns in some domains that are perturbations of a convex domain (see Figure 2b). The above proposition implies that such a stable pattern does not exist unless the nonlinearity has a large $C^{1}$ norm.

Theorem 1 involves the quantity $\lambda_{\gamma}$ which is rather implicit and depends on the solution itself. One may look for more explicit sufficient conditions for the non-existence of patterns.

The following result provides such a sufficient condition. It involves the stability of the solution, the geometry of the domain (through the Robin-curvature Laplacian) and supf'.

Theorem 2. Let $u$ be a pattern of (1). For any $a \geq 1$, we have

$$
(a-1) \lambda_{0}<\sup f^{\prime}-\mu_{a \gamma} .
$$

with $\mu_{a}$ from Definition 1. Consequently,

1. If $\mu_{\gamma} \geq \sup f^{\prime}$, there exists no (possibly unstable) pattern to (1). 


\section{If $\mu_{a \gamma} \geq \sup f^{\prime}$ for some $a \geq 1$, there exists no stable pattern to (1).}

This theorem provides sufficent conditions for the non-existence of patterns when the domain is not convex or when the patterns are not supposed to be stable. Therefore, this theorem covers many cases that are not covered by Theorem CHM.

Note that Theorem CHM is included in the above theorem as a particular case (except for the case of degenerate stability $\lambda_{0}=0$ ): if $\Omega$ is convex (i.e. $\gamma \geq 0$ ) and $u$ is a stable non-degenerate pattern (i.e. $\lambda_{0}>0$ ), then $\mu_{a \gamma}>0$, and since $a \mapsto \mu_{a \gamma}$ is nondecreasing, taking the limit $a \rightarrow+\infty$ in (5), we find a contradiction: hence $u$ is constant. On the contrary, if $\Omega$ is not convex (i.e. $\gamma \nsupseteq 0$ ), then $\lim _{a \rightarrow+\infty} \frac{\mu_{a \gamma}}{a}=-\infty([49])$, and the inequality (5] becomes trivial at the limit.

We point out that assertion 2. may not always improve assertion 1 . In dimension $n=2$, we have that $\int_{\partial \Omega} \gamma=2 \pi>0$ from the Gauss-Bonnet theorem, and we can show that $a \mapsto \mu_{a}$ reaches (a unique) positive maximum at some $a_{\star}>0$ (see 66. Theorem 2.1] with $g \equiv 0, h \equiv-\gamma$ ). However, we cannot guarantee that $a_{\star} \geq 1$ in general.

As a consequence of Theorem 2, we derive the non-existence of patterns if the domain is shrinking or if the nonlinearity is vanishing, when $\mu_{\gamma}>0$.

Corollary 1. Fix $(\Omega, f)$ such that $\mu_{\gamma}>0, \sup f^{\prime}<+\infty$, and set $0<\eta \leq$ $\sqrt{\frac{\mu_{\gamma}}{\sup f^{\prime}}}$. Then, there exists no (possibly unstable) solutions to (1) for $(\eta \Omega, f)$ or $\left(\Omega, \eta^{2} f\right)$, or more generally for any $\left(\eta^{1-\alpha} \Omega, \eta^{2 \alpha} f\right)$, with $\alpha \in(0,1)$.

Proof. The proof relies on the scaling property of $\mu_{\gamma}$. For $\eta>0$, denote $\gamma_{\eta}(\cdot):=$ $\eta^{-1} \gamma\left(\eta^{-1} \cdot\right)$ the minimal curvature of $\eta \Omega$ from Definition 1 Then, we have

$$
\mu_{\gamma_{\eta}}(\eta \Omega)=\eta^{-2} \mu_{\gamma}(\Omega)
$$

and so applying Theorem 2 proves the result.

Corollary 1 can be put into perspective with a theorem of Dancer that implies the non-existence of stable patterns in dilated domains, see Theorem 6 in Section 3

To apply our results to even more concrete situations, we need lower bounds on $\mu_{\gamma}$ in terms of classical geometry quantities (in-radius, curvature, etc). If the domain is convex, such a bound can be inferred from the literature on the Laplacian associated with standard positive Robin boundary condition (e.g. 8. 48, 61, 66]). For example, if $\Omega$ is convex then [61, Corollary 3] yields

$$
\mu_{a \gamma}>\frac{\pi^{2} a \underline{\gamma}}{4 R^{2} a \underline{\gamma}+\pi^{2} R}, \quad \forall a \geq 1,
$$

where $R$ is the in-radius of $\Omega$ and $\gamma=\inf _{\partial \Omega} \gamma$. Owing to this lower bound and Theorem 2, we can improve Theorem CHM in the following way. 
Corollary 2. Assume that $\Omega$ is a convex domain of in-radius $R$ and lowest curvature $\underline{\gamma}:=\inf _{\partial \Omega} \gamma$.

1. If $\sup f^{\prime}<\frac{\pi^{2} \underline{\gamma}}{4 \underline{\gamma} R^{2}+\pi^{2} R}$, there exists no (possibly unstable) pattern.

2. If $\frac{\pi^{2} \underline{\gamma}}{4 \underline{\underline{\gamma} R^{2}+\pi^{2} R}} \leq \sup f^{\prime}$, then, for $u$ a pattern of (1),

$$
\lambda_{0}<\sup f^{\prime}-\frac{2 \pi^{2} \underline{\gamma}}{8 R^{2} \underline{\gamma}+\pi^{2} R} .
$$

Proof. Plug (6) into (5), take $a=1$ for the first assertion and $a=2$ for the second assertion.

One can also improve (7) by taking the best value of $a$ in (5).

Corollary 2 improves the conclusion of Theorem CHM if $\sup f^{\prime}$ is not too large: the first assertion states the non-existence of all (possibly unstable) patterns if $\sup f^{\prime}$ is small enough, and the second assertion the non-existence of some unstable patterns if $\sup f^{\prime}$ is intermediate (note that the right member of $(7)$ is negative if $\sup f^{\prime}$ is close to $\left.\frac{\pi^{2} \underline{\gamma}}{4 \gamma R^{2}+\pi^{2} R}\right)$. However, if $\sup f^{\prime}$ is large this above result do not contain any relevant information since the right member in (7) is positive whereas Theorem CHM implies $\lambda_{0}<0$.

\subsection{Nonlinear Cacciopoli inequality}

We now propose a gradient estimate for the solutions of (1) which can be seen as a nonlinear Caccioppoli inequality, or an inverse Poincaré inquality. For the sake of generality, the result is stated for solutions of heterogeneous semi-linear equations, namely, when the nonlinearity $f=f(x, u)$ is allowed to depend on $x \in \Omega$.

Theorem 3. Let $u$ be a solution of

$$
\begin{cases}-\Delta u(x)=f(x, u(x)), & \forall x \in \Omega, \\ \partial_{\nu} u(x)=0, & \forall x \in \partial \Omega,\end{cases}
$$

where $f \in C^{1}(\Omega \times \mathbb{R}, \mathbb{R})$, and recall $\mu_{\gamma}$ from Definition 1 , Then

$$
\mu_{\gamma} \int_{\Omega}|\nabla u|^{2} \leq \int_{\Omega} f(x, u(x))^{2} d x
$$

with equality if and only if $u$ is constant.

We give a slight refinement of this result in Remark 1 (Section 5).

We emphasize that the theorem holds for possibly unstable solutions, and is universal in the sense that $\mu_{\gamma}$ does not depend on $f$. However, it is relevant 
only if the domain is such that the Robin Laplacian is positive (i.e. $\mu_{\gamma} \geq 0$ ). This class of domains contains the convex domains.

Multiplying (1) by $u$ and using the divergence theorem, we obtain the identity $\int_{\Omega}|\nabla u|^{2}=\int_{\Omega} u f(x, u)$, which allows to rewrite $(9)$ as

$$
\int_{\Omega} f(x, u)\left(f(x, u)-\mu_{\gamma} u\right) \geq 0 .
$$

Note also that a simple integration of (1) gives $\int_{\Omega} f(x, u)=0$, and thus $\int_{\Omega} f(x, u)^{2}$ is a $L^{2}$ measure of the variation of $f(x, u)$ around its means value.

As mentioned before, the inequality (9) can be seen as an inverse Poincaré inequality. Incidentally, it yields the upper bound

$$
\mu_{\gamma}<C_{P},
$$

where $C_{P}$ is the best constant for the second Poincaré inequality (or, equivalently, the inverse of the spectral gap of the Laplacian with Neumann boundary conditions). To prove this, simply take $f(x, u)=C_{P}^{-1} u$ and $u$ the eigenfunction associated with $C_{P}^{-1}$ in (9). This inequality implies that $\mu_{\gamma}$ is typically not too large in domains featuring a narrow bottleneck, such as Matano's dumbbell domains, introduced in Section 3 below.

\subsection{Flatness estimates}

The literature conveys the idea that stable patterns tend to be flat (i.e. onedimensional). This indeed holds in domains of the form $\mathbb{R} \times \omega$ or $\mathbb{R}^{2} \times \omega$ with $\omega \subset \mathbb{R}^{n-2}$ convex and bounded 54 . (but false in $\mathbb{R}^{n}$ when $n \geq 8$ [55]). Let us provide quantitative estimates on the flatness of patterns.

To begin with, let us refine Theorem 3 and infer a flatness estimate in terms of the spectral gap of the Robin-curvature Laplacian. The following result deals with the non-homogeneous problem (8). While Theorem 3 involves the lowest eigenvalue $\mu_{\gamma}$ of the Robin-curvature Laplacian, the following flatness estimate involves the second lowest eigenvalue $\mu_{\gamma, 2}$, defined as

$$
\mu_{\gamma, 2}:=\sup \left\{\inf \left\{\mathcal{G}_{\gamma}(\psi), \psi \in E,\|\psi\|_{L^{2}}=1\right\}, E \subset H^{1}(\Omega), \operatorname{dim} E=2\right\},
$$

with $\mathcal{G}_{\gamma}$ from Definition 1. We point out that, since $\mu_{\gamma}$ is associated with a unique (up to renormalization) eigenfunction $\varphi[30$, an equivalent definition is given by

$$
\mu_{\gamma, 2}:=\inf \left\{\mathcal{G}_{\gamma}(\psi), \psi \in H^{1}(\Omega),\|\psi\|_{L^{2}}=1, \int_{\Omega} \psi \varphi=0\right\}
$$

The fact that $\mu_{\gamma, 2}-\mu_{\gamma}>0$ is referred to in the literature as the spectral gap (in our context, we may call it the Robin-curvature spectral gap). Many estimates on the spectral gap for Neumann boundary conditions are available [2 22 23, 23, 57, and some of them can be adapted to the Robin-curvature boundary conditions using the method of [30]. 
Proposition 2. Let u be a pattern of the non-homogeneous equation (8). There exists a direction $e \in \mathbb{S}^{n-1}$ such that,

$$
\left(\mu_{\gamma, 2}-\mu_{\gamma}\right) \frac{\|\nabla u \cdot e\|^{2}}{\|\nabla u\|^{2}} \geq \mu_{\gamma, 2}-\sup f^{\prime}
$$

where $\|\cdot\|$ denotes the usual $L^{2}$-norm.

This proposition implies that patterns tend to be flat when $\mu_{\gamma, 2} \gg 1$. An analogous $k$-dimensional estimate can be derived with the $k$-ieth lowest eigenvalue of the Robin-curvature Lapacian, see Remark 2 (Section 6). We also point out that the estimate in Proposition 2 holds if we replace sup $f^{\prime}$ by $\frac{\int_{\Omega} f(u)^{2}}{\|\nabla u\|^{2}}$, see Remark 3

The following result is another flatness estimate for patterns of Morse index 1. For $u$ a solution of (1), we define $\lambda_{\gamma, 2}$ the second lowest eigenvalue of $\mathcal{F}_{\gamma}$ Definition 3 by

$$
\lambda_{\gamma, 2}:=\sup \left\{\inf \left\{\mathcal{F}_{\gamma}(\psi), \psi \in E,\|\psi\|_{L^{2}}=1\right\}, E \subset H^{1}(\Omega), \operatorname{dim} E=2\right\},
$$

Accordingly, we define $\lambda_{0,2}$ by replacing $\mathcal{F}_{\gamma}$ with $\mathcal{F}_{0}$ (Definition 2 in the above expression. We usually say that a solution of (1) is of Morse index 1 if $\lambda_{0}<0 \leq$ $\lambda_{0,2}$, that is, if there is only one direction of perturbation for which $u$ is unstable. This class of solutions is of particular importance, for example when considering solutions obtained from a mountain pass procedure or a minimization under constraint. Analgously, we say that a solution is of Robin-curvature Morse index 1 if $\lambda_{\gamma}<0 \leq \lambda_{\gamma, 2}$.

The following estimate is relevant for pattern of Morse index or Robincurvature Morse index 1.

Proposition 3. Let $u$ be a pattern of (1). There exists a direction $e \in \mathbb{S}^{n-1}$ such that

$$
1 \geq \frac{\|\nabla u \cdot e\|^{2}}{\|\nabla u\|^{2}} \geq \frac{\lambda_{\gamma, 2}}{\lambda_{\gamma, 2}-\lambda_{\gamma}}
$$

where $\|\cdot\|$ denotes the usual $L^{2}{ }^{2}$ norm.

In addition, there exists a direction $e \in \mathbb{S}^{n-1}$ such that, for any $a \geq 1$,

$$
(a-1)\left(\lambda_{0,2}-\lambda_{0}\right) \frac{\|\nabla u \cdot e\|^{2}}{\|\nabla u\|^{2}} \geq(a-1) \lambda_{0,2}+\mu_{a \gamma}-\sup f^{\prime} .
$$

This proposition is a refinement of Theorem 1 Indeed, if $u$ is Robincurvature-stable (i.e. $\lambda_{\gamma} \geq 0$ ), then (11) implies that $\frac{\lambda_{\gamma, 2}}{\lambda_{\gamma, 2}-\lambda_{\gamma}}=1$, and that $\nabla u=|\nabla u| e$, hence $u$ is one-dimensional; it is then easy to deduce that $u$ is constant.

Estimate (12) implies that patterns tend to be flat when $\lambda_{0,2}-\lambda_{0} \gg 1$. An analogous $k$-dimensional estimate can be derived for patterns of Morse index $k \leq n$, see Remark 2 (Section 6). 
Several refinements of the above flatness estimates are proposed in a series of remarks in Section 6 .

We also give another flatness estimate in Proposition 4 (Section 4.2 ) which relies on the geometric Poincaré inequality introduced by Sternberg and Zunbrum 64,65.

\subsection{Unbounded domains}

Let us now study whether our results extend to unbounded domains. We consider our main equation

$$
\begin{cases}-\Delta u(x)=f(u(x)), & \forall x \in \Omega, \\ \partial_{\nu} u(x)=0, & \forall x \in \partial \Omega, \\ u \in C^{3}(\bar{\Omega}), \quad \nabla u \in L^{\infty}(\Omega) & \end{cases}
$$

where the domain $\Omega \subset \mathbb{R}^{n}(n \geq 2)$ is possibly unbounded and uniformly $C^{2}$, and, as before, $f$ is $C^{1}$.

If $u$ is a solution of (13), the definition of Robin-curvature-stability through the sign of $\lambda_{\gamma}$ in Definition 3 is not licit since $f^{\prime}(u)$ may not be bounded and therefore $f^{\prime}(u) \psi^{2}$ may not be integrable for a generic test function $\psi \in H^{1}$. Instead, when the domain is unbounded, we define $\lambda_{\gamma}$ on the truncated domains

$$
\lambda_{\gamma}^{R}:=\inf _{\substack{\psi \in H^{1}\left(\Omega_{R}\right) \cap H_{0}^{1}\left(B_{R}\right) \\\|\psi\|_{L^{2}}=1}} \mathcal{F}_{\gamma}(\psi), \quad \forall R>0,
$$

where $\Omega_{R}:=\Omega \cap B_{R}$ and $B_{R}$ is the ball of radius $R$ centered at the origin. Here, the infimum is taken over functions $\psi \in H^{1}\left(\Omega_{R}\right)$ whose support is included in $B_{R}$. The fact that we impose Dirichlet boundary conditions on $\Omega \cap \partial B_{R}$ ensures that $R \mapsto \lambda_{R}$ is decreasing. We then define

$$
\lambda_{\gamma}:=\lim _{R \rightarrow+\infty} \lambda_{\gamma}^{R}, \quad \text { and } \quad \lambda_{0}:=\lambda_{\left.\gamma\right|_{\gamma \equiv 0}}=\lim _{R \rightarrow+\infty} \lambda_{0}^{R} .
$$

From the dominated convergence theorem, we can prove that the above definitions of $\lambda_{\gamma}$ and $\lambda_{0}$ coincide with definitions (2) and (3) as long as $f^{\prime}(u)$ is bounded (in particular, if the domain is bounded or if the solution $u$ is bounded).

It will be understood without further notice that when the domain is unbounded, $\lambda_{\gamma}$ and $\lambda_{0}$ are defined through (15). By convention, we set $\gamma=0$ if $\Omega=\mathbb{R}^{n}$.

The classification of solutions in unbounded domains is a very active topic that is related to many fundamental problems. A vast literature is devoted to De Giorgi's conjecture about the classification of stable patterns in the particular case of the entire space $\Omega=\mathbb{R}^{n}$ and the Allen-Cahn nonlinearity $f(u)=u-u^{3}$ :

it is known that bounded stable patterns are necessarily planar in dimensions $n=1,2[11$, and that it is not true in dimensions $n \geq 8$ [55]. The question 
remains open for the intermediate dimensions $3 \leq n \leq 7$. We refer the reader to 69] for a state of the art on this question and De Giorgi's conjecture.

In a previous paper [54], we studied whether Theorem CHM] extends to unbounded domains. We showed that, if the domain is convex and possibly unbounded, there exist no patterns to 13 which are stable non-degenerate (i.e., with $\lambda_{0}>0$ ). We also gave a classification of possibly degenerate stable solutions (i.e., with $\lambda_{0} \geq 0$ ) if the domain is convex and further satisfies the growth condition at infinity

$$
|\Omega \cap\{|x| \leq R\}|=O\left(R^{2}\right), \quad \text { when } R \rightarrow+\infty .
$$

The results contained in [54] extends the well known one-dimensional symmetry of stable patterns in $\mathbb{R}^{2}$ to any convex domain satisfying (16). More precisely, under assumption (16), if the domain is convex and is not a straight cylinder (i.e., the domain is not of the form $\Omega=\mathbb{R} \times \omega$, with $\omega \subset \mathbb{R}^{n-1}$ ), then there exists no patterns to $(13)$. If the domain is a convex straight cylinder, stable solutions are either constant or monotonic planar solutions which, if bounded, connect two stable roots $\left(z_{1}, z_{2}\right)$ of $f$ such that $\int_{z_{1}}^{z_{2}} f=0$.

We emphasize that none of the above conclusions hold for solutions with a unbounded gradient: indeed, $u(x):=e^{x}$ is a solution of $-u^{\prime \prime}=-u$ in $\mathbb{R}$ for which $\lambda_{0}>0$. It justifies why we assume a priori in (13) that the solution $u$ has a bounded gradient.

The present article improves the results of [54] and thus extends some results on De Giorgi's conjecture (namely the one-dimensional symmetry of stable patterns in $\mathbb{R}^{2}$ ) to a larger class of domains.

In view of Theorem 1] it is reasonnable to expect that the results of [54], which hold for stable solutions in convex domains, remain true for Robincurvature-stable solutions in any (possibly non-convex) domain. This is what we state in Theorem 4 below.

The following result extends Theorem 1 to unbounded domains under either a non-degeneracy assumption on the Robin-curvature-stability, or a control on the growth of the domain at infinity.

Theorem 4. Let $u$ be a solution of (13) and assume that $u$ is Robin-curvaturestable (i.e. $\lambda_{\gamma} \geq 0$ ).

1. If the Robin-curvature-stability of $u$ is not too degenerate, i.e.,

$$
\liminf _{R \rightarrow+\infty} R^{2} \lambda_{\gamma}^{R}=+\infty
$$

(in particular, if $\lambda_{\gamma}>0$ ), then $u$ is constant.

2. If $\Omega$ satisfies the growth condition at infinity $(16)$ and is not a straight cylinder (i.e. $\Omega$ is not of the form $\mathbb{R} \times \omega$ with $\omega \subset \mathbb{R}^{n-1}$ ), then $u$ is constant. 
3. If $\Omega=\mathbb{R} \times \omega$ satisfies the growth condition at infinity (16), then $u$ is either constant, or is a monotonic planar solution. If, in addition, $u$ is bounded then it connects two stable roots $\left(z_{1}, z_{2}\right)$ of $f$ such that $\int_{z_{1}}^{z_{2}} f=0$.

We recall that in a convex domain, owing to (4), stability implies Robincurvature-stability. The above theorem therefore contains the results of [54] as special cases.

Assumption (17) in the first assertion of Theorem 4 encompasses the case of nondegenerate Robin-curvature-stability $\lambda_{\gamma}>0$. Therefore, this result improves [54, Theorem 1.4] which only deals with the case of nondegenerate stability $\left(\lambda_{0}>0\right)$.

The second and third assertions of the above theorem give a complete classification of Robin-curvature-stable patterns in domains satisfying (16). This is a refinement of [54. Theorem 1.5] which deals with stable solutions in convex domains. We point out that assumption $(16)$ is satisfied by any subset of $\mathbb{R}^{2}$ and also by some subsets of $\mathbb{R}^{n}, n>2$, which size do not grow too fast at infinity.

If the domain is a straight cylinder, then $\gamma \leq 0$, and so $\lambda_{\gamma} \leq \lambda_{0}$ : in this case, Robin-curvature-stability implies stability. If the domain is a convex straight cylinder, then $\lambda_{\gamma}=\lambda_{0}$ and the third assertion in Theorem 4 coincides with [54. Theorem 1.5 2.]. Bounded planar patterns might exist in such domains. For example, the Allen-Cahn equation in $\mathbb{R},-u^{\prime \prime}=u\left(1-u^{2}\right)$ admits the explicit solution $u: x \mapsto \tanh \frac{x}{\sqrt{2}}$ which is stable degenerate (i.e. $\lambda_{\gamma}=\lambda_{0}=0$ ).

The restriction (17) on the growth of the domain at infinity is reminiscent of numerous previous works on similar topics and is believed to be sharp, see 54 for more details. In [54] we asked whether an assumption of strict convexity of the domain allows to relax (17) for the non-existence of stable patterns. Unfortunately, Theorem 4 does not provide a positive answer to this question, because we may not have $\lambda_{\gamma}>\lambda_{0}$ in general but only $\lambda_{\gamma} \geq \lambda_{0}$, even if the domain is strictly convex.

Let us give more details. For $\alpha \geq 0$, consider the principal eigenvalue of the $\alpha$-Robin Laplacian

$$
\mu_{\alpha}:=\inf _{\substack{\psi \in H^{1}(\Omega) \\\|\psi\|_{L^{2}}=1}} \int_{\Omega}|\nabla \psi|^{2}+\alpha \int_{\partial \Omega} \psi^{2} .
$$

If the domain is bounded, it is classical that $\alpha \mapsto \mu_{\alpha}$ is strictly increasing (see e.g. the last part of the proof of Lemma 3). If the domain is unbounded, we still have that $\alpha \mapsto \mu_{\alpha}$ is nondecreasing, however, the strict monotonicity does not hold in general. Indeed, for $\Omega=(0,+\infty)$, we have $\mu_{\alpha}=0$ for all $\alpha \geq 0$. To prove this, notice that the function $\psi(x)=\alpha x+1$ is positive on $[0,+\infty)$ and satisfies $\Delta \varphi=0$ and $-\varphi^{\prime}(0)+\alpha \varphi(0)=0$; hence $\varphi$ is a positive supersolution of the Robin Laplacian in $\Omega$, which classically implies $\mu_{\alpha} \leq 0$.

The fact that $\alpha \mapsto \mu_{\alpha}$ is not strictly monotonic suggests that, even if we assume that the domain is strictly convex (i.e. $\gamma>0$ ), we cannot guarantee that the strict inequality $\lambda_{\gamma}>\lambda_{0}$ holds in general, contrarily to what is stated 
in Lemma 3 for bounded domains. Hence, even in a strictly convex domain $(\gamma>0)$, a stable degenerate pattern $\left(\lambda_{0}=0\right)$ can have a degenerate Robincurvature-stability (i.e. $\lambda_{\gamma}=0$ ).

The following result states that Theorem 2 holds in unbounded domain.

Corollary 3. Let $u$ be a pattern of 13 . For any $a \geq 1$, we have that

$$
(a-1) \lambda_{0} \leq \sup f^{\prime}-\mu_{a \gamma},
$$

with $\mu_{a}$ from Definition 1. Consequently,

1. If $\mu_{\gamma}>\sup f^{\prime}$, there exists no (possibly unstable) pattern to 13 .

2. If $\mu_{a \gamma}>\sup f^{\prime}$ for some $a \geq 1$, there exists no stable pattern to (13).

This gives a criterion for the non-existence of patterns in unbounded domains, involving the stability of the solution, the geometry of the domain, and the $C^{1}$ norm of the nonlinearity.

We emphasize that (18) features a large inequality, whereas (5) features a strict inequality. Accordingly, the condition for the non-existence of pattern in the above theorem involves a large inequality, contrarily to Theorem 2. In fact, sufficient conditions for the non-existence of patterns are

$$
\liminf _{R \rightarrow+\infty} R^{2}\left(\mu_{\gamma}-\sup f^{\prime}\right)>0
$$

or

$$
\mu_{\gamma} \geq \sup f^{\prime} \text { and } \Omega \text { satisfies } 16 \text {. }
$$

Let us now adapt Theorem 3 to unbounded domains. In this context, $\nabla u$ and $f(x, u(x))$ may not belong to $L^{2}$. We thus have to consider a cut-off function: for $R>0$ and $\delta>0$, set

$$
\chi_{R}(x):=\chi\left(\frac{|x|}{R}\right), \quad \forall x \in \mathbb{R}^{n},
$$

with $\chi$ a smooth nonnegative function such that

$$
\chi(z)=\left\{\begin{array}{ll}
1 & \text { if } 0 \leq z \leq 1 \\
0 & \text { if } z \geq 2
\end{array}, \quad\left|\chi^{\prime}\right| \leq 2 .\right.
$$

Let us also define $\mu_{\gamma}^{R}$ the principal eigenvalue of the Robin-curvature Laplacien on the truncated domain $\Omega_{R}:=\Omega \cap B_{R}$ as

$$
\mu_{\gamma}^{R}:=\inf _{\substack{\psi \in H^{1}\left(\Omega_{R}\right) \cap H_{0}^{1}\left(B_{R}\right) \\\|\psi\|_{L^{2}}=1}} \mathcal{G}_{\gamma}(\psi), \quad \forall R>0 .
$$


Theorem 5. Let $u$ be a solution of

$$
\begin{cases}-\Delta u(x)=f(x, u(x)), & \forall x \in \Omega, \\ \partial_{\nu} u(x)=0, & \forall x \in \partial \Omega, \\ u \in C^{3}(\bar{\Omega}), \quad \nabla u \in L^{\infty}(\Omega), & \end{cases}
$$

where $f \in C^{1}(\Omega \times \mathbb{R}, \mathbb{R})$ and $\Omega$ is not necessarily bounded. Then

$$
\mu_{\gamma}^{R} \int_{\Omega} \chi_{R}^{2}|\nabla u|^{2} \leq \int_{\Omega}\left(\left|\nabla \chi_{R}\right||\nabla u|+\chi_{R} f(x, u)\right)^{2} d x .
$$

Compared to Theorem 3 the right hand-side of 21) features an additionnal term $\left|\nabla \chi_{R}\right||\nabla u|$ which is due to the truncation. The following corollary states that this term can somehow be neglected when $R \rightarrow+\infty$.

Corollary 4. Under the same conditions, if $u$ is not constant, then

$$
\limsup _{R \rightarrow+\infty} \frac{\int_{\Omega} \chi_{R}^{2} f(x, u)^{2} d x}{\int_{\Omega} \chi_{R}^{2}|\nabla u|^{2}} \geq \frac{\mu_{\gamma}}{2} .
$$

The flatness estimates stated in Proposition 2 and Proposition 3 formally hold in unbounded domains. However, there is no spectral gap in unbounded domains (not even for the Laplace operator in $\mathbb{R}$ ) and therefore these results are not relevant.

\section{Related literature}

In this section, we present the main known results in the literature on the existence, non-existence, and qualitative properties of patterns, and we discuss the implications of our results.

Existence of stable patterns in dumbbell domains. In his pioneering article 50], Matano constructs a counterexample to Theorem CHM by proving that (1) admits stable patterns for some $(\Omega, f)$. The author considers a generic bistable nonlinearity, and a so-called dumbbell domain, which consists in two disjointed convex sets connected by a bottleneck of width $\varepsilon \ll 1$, see Figure 1 . He proves the existence of a stable pattern if $\varepsilon$ is small enough (and provides a quantitative estimate on the smallness of $\varepsilon$ ). This construction has been simplified and extended in many directions $[7,25,40,43,45]$, and a general theoretical framework to deal with dumbbell domains is provided in [3 5].

Matano's construction is based on a variational argument and relies on Poincaré's second inequality. The best constant in the second Poincaré inequality, denoted by $C_{P}$, somehow measures the connectivity of the domain $\left(C_{P}\right.$ is also the inverse of the spectral gap of the Laplacian with Neumann boundary conditions). Heuristically, in dumbbells domains, the presence of the bottleneck hinders the connectivity of the domain and so the second Poincaré inequality is 


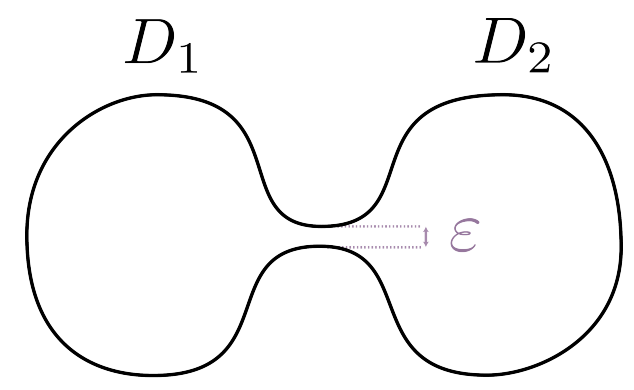

Figure 1: dumbbell domain

typically very weak, i.e., $C_{P} \ll 1$. Inequality $(10)$ implies that $\mu_{\gamma}$ is also typically very small. We point out that Theorem 2 contains a somehow converse statement since it guarantees the non-existence of patterns if $\mu_{\gamma}$ is large enough (compared to the $C^{1}$ norm of the nonlinearity).

We emphasize that the existence of stable patterns relies more on the presence of a bottleneck (which must be very narrow compared to $f$ ) than on the non-convexity of the domain. This can be seen in dimension $n=2$ by the following result, obtained after a simple rescaling from a result of Dancer.

Theorem 6 (Theorem 5 in [26]). Let $\Omega \subset \mathbb{R}^{2}$ be a smooth domain, and assume $0 \in \Omega$. Further assume that $f$ satisfies

$$
\left\{\begin{array}{c}
\exists(M, m) \in \mathbb{R}^{2} \text { such that }\left\{\begin{array}{l}
f>0 \text { on }(-\infty, m), \\
f<0 \text { on }(M,+\infty) .
\end{array}\right. \\
\text { the stable roots of } f \text {, denoted }\left(z_{i}\right) \text { are simple and isolated, } \\
\forall z_{i} \neq z_{j}, \quad \int_{z_{i}}^{z_{j}} f \neq 0 .
\end{array}\right.
$$

Then, there exists no stable pattern to (1) in the dilated domain $\varepsilon^{-1} \Omega$, if $\varepsilon \ll 1$.

This theorem can be put into perspective with Corollary 1 which state the non-existence of patterns in shrinking domains.

Link with minimal surfaces. Consider equation (1) with the rescaled AllenCahn nonlinearity $f_{\varepsilon}(u):=\frac{1}{\varepsilon}\left(u-u^{3}\right)$. This equation arises in the Van der WaalCahn-Hiliard model for phase transitions [19]. A series of pioneering papers [18, 51, 52, 60 establishes that when $\varepsilon \rightarrow 0$, any level set of a sequence of stable solutions $u_{\varepsilon}$ converges to a set $E$ which is a local minimizer of the perimeter functional in $\Omega$ (in other words, $\partial E \cap \Omega$ is a minimal surface in $\Omega$ ).

Conversely, Kohn and Sternberg [47] show that, given such a set $E$ which minimizes the perimeter functional in $\Omega$, there exists a sequence of stable solutions $u_{\varepsilon}$ converges to $\mathbb{1}_{E}-\mathbb{1}_{\Omega \backslash E}$. Thus, provided there exists such a (non-trivial) set $E$, it proves that there exists a stable pattern to (1) for $f_{\varepsilon}, \varepsilon \ll 1$. A typical example is the dumbbell domain, with $\partial E$ located at the bottleneck of the 
domain (Figure 2a); thus Kohn and Stenberg's pattern echoes Matano's. On the contrary, if the domain $\Omega$ is a convex domain, there exists no such set $E$; accordingly, from Theorem CHM there exists no stable pattern as well.

As mentioned in the introduction, the result of Kohn and Sternberg allows constructing stable patterns in star-shaped domains obtained as a small perturbation of convex domains, see Figure 2b. In dimension $n \geq 3$, such a domain can also be chosen with positive mean curvature. In that sense, the convexity assumption in Theorem CHM is sharp.

Nevertheless, we see from Proposition 1 that, when the domain is close from being convex, this construction can only be achieved when $\varepsilon \ll 1$, i.e., when the nonlinearity has a large $C^{1}$ norm.

We also emphasize that, contrary to Matano's pattern, Kohn and Sternberg's construction relies on the fact that the Allen-Cahn nonlinearity is balanced (i.e. $\left.\int_{-1}^{+1} f=0\right)$. This is indeed a necessary condition, as can be seen in dimension $n=2$ by Theorem 6 which, after a simple rescaling, implies the non-existence of patterns in any domain for unbalanced nonlinearity of large $C^{1}$ norm.
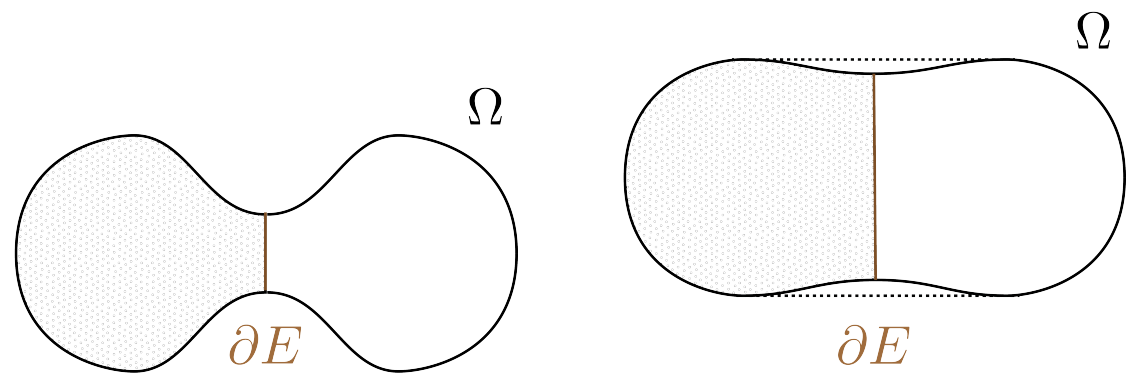

(a) dumbbell domain

(b) star-shaped domain obtained as a small perturbation of a convex domain

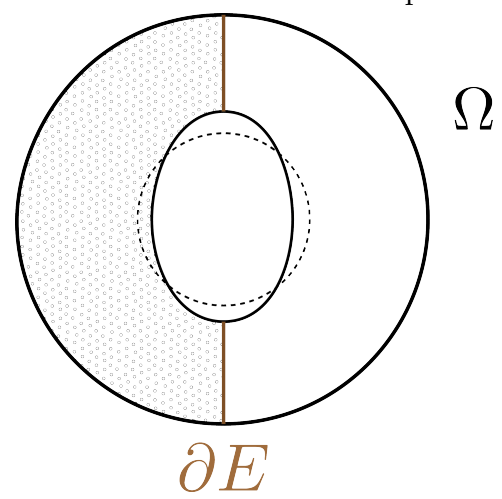

(c) Small perturbation of an annulus

Figure 2: Domains $\Omega$ admitting patterns for $f=\frac{1}{\varepsilon^{2}}\left(u-u^{3}\right), \varepsilon \ll 1$. The dotted area $E$ stands for a set with minimal perimeter in $\Omega$. 
Angular symmetry. Matano 50] also shows that, if the domain is a solid of revolution, stable patterns must be invariant with respect to rotations around the axis of revolution. If, in addition, the section is convex, there exists no stable pattern. In particular, it proves the non-existence of stable pattern for any $C^{1}$ nonlinearity in certain non-convex domains, such as torus or rings.

However, it has to be noticed that this result on the non-existence of stable pattern is not robust under small perturbation of the domain since patterns can be constructed with the method of Kohn and Sternberg in domains as in Figure 2c

Matano's angular symmetry suggests that stable patterns inherit some symmetries of the domain. It is also true for invariance by translations: stable patterns in $\mathbb{R} \times \omega$ (where $\omega \subset \mathbb{R}^{n-1}$ is bounded) are invariant with respect to $x_{1}$ 54. Nevertheless, the symmetries by rotation and translation are the only ones to be expected, since Cartesian and angular differentiations are the only first-order differential operators commuting with the Laplacian. It is, however, natural to consider this question for patterns on manifolds. This has been done by many authors $7,36,37,41,45,56,59$.

Propagation in reaction-diffusion equations. Many papers deal with the spreading properties of reaction-diffusion equations, which are the evolution equations associated with (1). In this context, the existence of stable patterns corresponds to the possibility of the blocage of an invading wave; on the other hand, the non-existence of stable patterns means that there is a complete invasion.

Let us briefly report on the article of Berestycki, Hamel and Matano 12 which studies the propagation in the presence of an obstacle. Consider an exterior domain $\Omega=\mathbb{R}^{n} \backslash K$, where the obstacle $K \subset \mathbb{R}^{n}$ is compact, and a bistable unbalanced nonlinearity, say $f(u)=u(1-u)(u-a)$, with $a<1 / 2$, so that $\int_{0}^{1} f>0$. This last inequality (which is not satisfied by the Allen-Cahn nonlinearity) ensures that any traveling wave connecting 0 to 1 converges to 1 in large times (roughly speaking " 1 invades 0 "). The authors consider the special class of solution to (1) which satisfies

$$
\lim _{|x| \rightarrow+\infty} u(x)=1 .
$$

This class of solution is obtained as the limit in large times of a generalized traveling wave.

The authors in $[12$ give the following results. On the one hand, if the obstacle features a bottleneck (as in a dumbbell domain), there exist stable patterns satisfying (22). On the other hand, if the obstacle is star-shaped or axially convex, there exists no (possibly unstable) pattern satisfying (22). We point out, however, that in such domains (see Figure 3) there might exist stable patterns which do not satisfy $(22)$ but satisfy $\lim _{|x| \rightarrow+\infty} u(x)=0$. See also 10 for similar conclusions if the domain is a cylinder with varying cross section, and 14,15 for the case of non-local diffusion. 


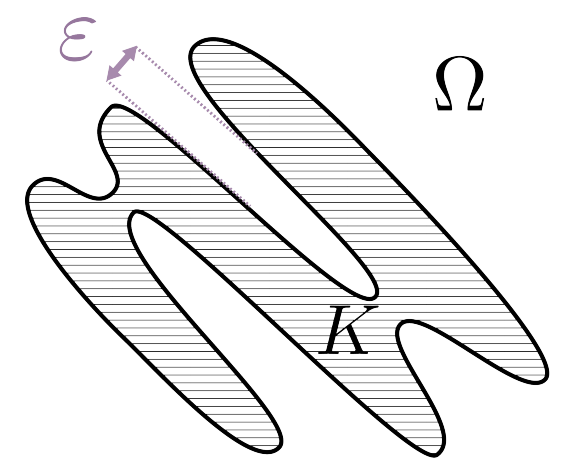

Figure 3: Example of a domain $\Omega=\mathbb{R}^{n} \backslash K$, exterior of an axially convex obstacle, which does not admit any pattern satisfying (22), but admits stable patterns (for some $f$ ).

Further references. Many authors have also been interested in understanding how the existence of stable patterns could emerge from the non-homogeneity of coefficients, for example, under non-homogeneous diffusivity $34,63,70$ or non-homogeneous reaction 1, 16,62 .

Finally, we mention that the regularity of weak stable solutions has been widely studied, see [17,35], and references therein. See also 21] for properties on the level sets of stable patterns in the case of Dirichlet boundary conditions. In these works, it appears that the assumption on the convexity of the domain is central.

\section{Non-existence of patterns}

\subsection{Proof of Theorem 1}

We give a proof which is in the spirit of [20,50]; we propose an alternative proof in Section 4.2 We begin with a classical geometric lemma which was first stated in 20,50 (in a slightly less general form).

Lemma 1 ( [20,50]). Let $\Omega \subset \mathbb{R}^{n}$ be a smooth domain and recall $\gamma(\cdot)$ from Definition 1. Let u be a $C^{2}$ function such that

$$
\partial_{\nu} u=0 \quad \text { on } \partial \Omega .
$$

Then,

$$
\frac{1}{2} \partial_{\nu}|\nabla u|^{2}=-\nabla u \cdot \nabla \nu \cdot \nabla u \leq-\gamma|\nabla u|^{2} \quad \text { on } \partial \Omega,
$$

with equality if $n=2$. 
Proof. On the one hand, differentiating (23) with respect to the vector field $\nabla u$ (note that $\nabla u$ is tangential to $\partial \Omega$ ) leads to

$$
0=\nabla \partial_{\nu} u \cdot \nabla u=\nabla u \cdot \nabla^{2} u \cdot \nu+\nabla u \cdot \nabla \nu \cdot \nabla u .
$$

On the other hand, we have

$$
\partial_{\nu}|\nabla u|^{2}=\nabla\left(|\nabla u|^{2}\right) \cdot \nu=2 \nabla u \cdot \nabla^{2} u \cdot \nu .
$$

Using (24) in the above expression, we deduce

$$
\partial_{\nu}|\nabla u|^{2}=-2 \nabla u \cdot \nabla \nu \cdot \nabla u .
$$

We recall that $\gamma(x)$ is defined as the lowest eigenvalue of $\nu(x)$ restricted to the tangent space $\nu(x)^{\perp}$, i.e., by the Rayleigh quotient formula,

$$
\gamma(x)=\inf _{X \in \nu(x)^{\perp} \backslash\{0\}} \frac{X \cdot \nabla \nu(x) \cdot X}{|X|^{2}}, \quad \forall x \in \partial \Omega .
$$

Therefore, $\nabla u \cdot \nabla \nu \cdot \nabla u \geq \gamma|\nabla u|^{2}$, with equality if $n=2$. From this and (25), we conclude the proof.

Now, we give the following intermediate result.

Lemma 2. Let $u$ be a solution of (1), and set $v_{i}:=\partial_{x_{i}} u$, for all $i \in\{1, \ldots, n\}$. We have that

$$
\sum_{i=1}^{n} \mathcal{F}_{\gamma}\left(v_{i}\right) \leq 0
$$

with $\mathcal{F}_{\gamma}$ from Definition 3.

Proof. Differentiating (1) with respect to $x_{i}$, we find that $v_{i}:=\partial_{x_{i}} u$ satisfies the linearized equation

$$
-\Delta v_{i}-f^{\prime}(u) v_{i}=0 \quad \text { in } \Omega .
$$

Multiplying by $v_{i}$ and using the divergence theorem, we find

$$
\int_{\Omega}\left|\nabla v_{i}\right|^{2}-\int_{\Omega} f^{\prime}(u) v_{i}^{2}=\int_{\partial \Omega} v_{i} \partial_{\nu} v_{i}
$$

and so, writing $v_{i} \partial_{\nu} v_{i}=\frac{1}{2} \partial_{\nu} v_{i}^{2}$, we obtain

$$
\mathcal{F}_{\gamma}\left(v_{i}\right)=\int_{\partial \Omega} \frac{1}{2} \partial_{\nu} v_{i}^{2}+\int_{\partial \Omega} \gamma v_{i}^{2} .
$$

Summing the above equation for $i \in\{1, \ldots, n\}$ and using that $|\nabla u|^{2}=v_{1}^{2}+$ $\cdots+v_{n}^{2}$ gives

$$
\sum_{i=1}^{n} \mathcal{F}_{\gamma}\left(v_{i}\right)=\int_{\partial \Omega} \frac{1}{2} \partial_{\nu}|\nabla u|^{2}+\int_{\partial \Omega} \gamma|\nabla u|^{2} .
$$

The right-hand side of the above identity is nonpositive from Lemma 1, which achieves the proof. 
With these lemmas in hands, we are ready to prove Theorem 1 . Let $u$ be a solution of (1) and assume $\lambda_{\gamma} \geq 0$. Set $v_{i}:=\partial_{x_{i}} u$, for all $i \in\{1, \ldots, n\}$. Since $\lambda_{\gamma} \geq 0$, we have that $\mathcal{F}_{\gamma}(\cdot) \geq 0$, hence, using Lemma 2 ,

$$
0 \leq \mathcal{F}_{\gamma}\left(v_{i}\right) \leq \sum_{k=1}^{n} \mathcal{F}_{\gamma}\left(v_{k}\right)=\int_{\partial \Omega} \frac{1}{2} \partial_{\nu}|\nabla u|^{2}+\int_{\partial \Omega} \gamma|\nabla u|^{2} \leq 0 .
$$

We infer that $\mathcal{F}_{\gamma}\left(v_{i}\right)=0$, that $\lambda_{\gamma}=0$, and that $v_{i}$ minimizes $\mathcal{F}_{\gamma}$.

It is then classical that $v_{i}$ must have a constant strict sign on $\bar{\Omega}$ (more precisely, $v_{i}$ must be a constant multiple of the eigenfunction associated with $\lambda_{\gamma}[30]$. Now, from $\partial_{\nu} u=0$ on the closed surface $\partial \Omega$, we deduce that $v_{i}$ vanishes on some point of the boundary. Since $v_{i}$ has a constant strict sign, we deduce that $v_{i} \equiv 0$, which completes the proof.

\subsection{Alternative proof of Theorem 1}

The proof of Theorem 1 presented in Section 4.1 relies on Lemma 2 which involves the term $\sum_{i=1}^{n} \mathcal{F}_{\gamma}\left(v_{i}\right)$. However, it is not easy to give an intuitive interpretation of this quantity, and although the method is simple, it seems ad hoc. Let us give an alternative proof where the above quantity appears naturally. The idea is inspired by the famous geometric Poincaré inequality introduced by Sternberg and Zunbrum $[64,65$, and consists of using $v=|\nabla u|$ as a test function in $\mathcal{F}_{\gamma}$.

In fact, we are going to prove a more precise result than Theorem 1 .

Proposition 4. Let $u$ be a nonconstant solution of (1). Then,

$$
\lambda_{\gamma} \leq \frac{\|\nabla|\nabla u|\|^{2}-\left\|\nabla^{2} u\right\|^{2}}{\|\nabla u\|^{2}}<0
$$

where $\|\cdot\|$ denotes the usual $L^{2}$-norm and $\nabla^{2} u$ is the Hessian matrix of $u$.

Proof. Set $v_{i}=\partial_{x_{i}} u$ for all $i=1, \ldots, n$ and $v=|\nabla u|=\sqrt{v_{1}^{2}+\cdots+v_{n}^{2}}$. A straightforward computation gives (on the set $\{v \neq 0\}$ )

$$
-\Delta v-f^{\prime}(u) v=\frac{1}{v}\left(|\nabla v|^{2}-\sum_{i=0}^{n}\left|\nabla v_{i}\right|^{2}\right) \leq 0 .
$$

Multiplying by $v$ and using the divergence theorem, we find

$$
-\int_{\partial \Omega} v \partial_{\nu} v+\int_{\Omega}|\nabla v|^{2}-\int_{\Omega} f^{\prime}(u) v^{2}=\int_{\Omega}\left[|\nabla v|^{2}-\sum_{i=0}^{n}\left|\nabla v_{i}\right|^{2}\right] .
$$

Writing $v \partial_{\nu} v=\frac{1}{2} \partial_{\nu} v^{2}$, and using Lemma 1, we obtain

$$
\mathcal{F}_{\gamma}(v) \leq \int_{\Omega}\left[|\nabla v|^{2}-\sum_{i=0}^{n}\left|\nabla v_{i}\right|^{2}\right]
$$


From the definition of $\lambda_{\gamma}$ Definition 3, we have that $\mathcal{F}_{\gamma}(v) \geq \lambda_{\gamma}\|v\|^{2}$. We conclude the proof by using this inequality and that $\int_{\Omega} \sum_{i=0}^{n}\left|\nabla v_{i}\right|^{2}=\left\|\nabla^{2} u\right\|$ in 28 .

We emphasize that (28) is simply a rewriting of (26) (we recall that $v^{2}=$ $\left.v_{1}^{2}+\cdots+v_{n}^{2}\right)$. The advantage of this formulation is that we can give a a geometric interpretation of the right member: as stated in [65. Lemma 2.1],

$$
|\nabla v|^{2}-\sum_{i=0}^{n}\left|\nabla v_{i}\right|^{2}= \begin{cases}|\nabla u|^{2} \sum_{i=1}^{n-1} \kappa_{i}^{2}+\left|\nabla_{T}\right| \nabla u||^{2} & \text { on }\{\nabla u \neq 0\}, \\ 0 & \text { a.e. on }\{\nabla u=0\}\end{cases}
$$

where $\kappa_{i}$ are the principal curvature of the level set of $u$ and $\nabla_{T}$ denotes the differentiation which is tangent to this level set.

In particular, this term is nonpositive and is identically zero if and only if $u$ is one-dimensional. It yields a simple proof of Theorem 1 ; if $\lambda_{\gamma} \geq 0$, we deduce from (28) and the above geometric interpretation that $u$ is one-dimmensional, and then we easily conlcude that $u$ is constant.

For $u$ a pattern of (1), inequality (27) provides a negative upper bound on $\lambda_{\gamma}$ (whereas Theorem 1 only implies $\lambda_{\gamma}<0$ ). This inequality can also be seen as a flatness estimate.

\subsection{Proof of Proposition 1}

We begin with an intermediate result stating that the strict inequality holds in 4 .

Lemma 3. Assume that $\Omega$ is bounded and convex, and let $u$ be a solution of (1). Then,

$$
\lambda_{\gamma}>\lambda_{0}
$$

with $\lambda_{0} \lambda_{\gamma}$ from Definition 2 and Definition 3.

Proof. First, let us prove that there exists a point $x_{0} \in \partial \Omega$ at which $\gamma\left(x_{0}\right)>0$. Since $\Omega$ is bounded, the norm mapping $x \in \Omega \mapsto|x|$ reaches its maximum $R>0$ at some point $x_{0} \in \partial \Omega$. Denoting by $B_{R}$ the ball of radius $R$ centered at the origin, we have that $\Omega \subset B_{R}$ and that $\Omega$ and $B_{R}$ are tangent at $x_{0}$. From a standard comparison principle, we deduce that $\gamma\left(x_{0}\right) \geq \frac{1}{R_{0}}>0$.

Then, consider $\varphi$ the (unique) positive eigenfunction associated with $\lambda_{\gamma}$ (given, for example, by $[30]$ ). It is classical that $\varphi>0$ in $\bar{\Omega}$. From this and the fact that $\gamma\left(x_{0}\right)>0$, we infer $\int_{\partial \Omega} \gamma \varphi^{2}>0$. It implies

$$
\lambda_{\gamma}=\mathcal{F}_{\gamma}(\varphi)=\mathcal{F}(\varphi)+\int_{\partial \Omega} \gamma \varphi^{2}>\mathcal{F}_{0}(\varphi) \geq \lambda_{0},
$$

which concludes the proof. 
Now, let us prove Proposition 1 Assume that $\Omega$ is convex and let $\left(\Omega_{\varepsilon}, f_{\varepsilon}\right)$, $\varepsilon>0$ be a family of smooth perturbations which converges to $(\Omega, f)$ as $\varepsilon \rightarrow 0$. By contradiction, assume that there exists $u_{\varepsilon}$ a sequence of stable patterns of (1) with $\left(\Omega_{\varepsilon}, f_{\varepsilon}\right)$. We denote $\lambda_{0, \varepsilon}$ and $\lambda_{\gamma, \varepsilon}$ the quantities from (2) and (3) corresponding to $u_{\varepsilon}$. Since $u_{\varepsilon}$ is a stable pattern, we have $\lambda_{0, \varepsilon} \geq 0$ (by the definition of stability) and $\lambda_{\gamma, \varepsilon}<0$ (from Theorem 1), which implies

$$
\lambda_{\gamma, \varepsilon}<\lambda_{0, \varepsilon} .
$$

From classical elliptic estimates, $u_{\varepsilon}$ is bounded in $C^{2, \alpha}\left(\bar{\Omega}_{\varepsilon}\right)$, uniformly in $\varepsilon>0$ (see e.g. [39, Theorem 6.30]). Up to extraction of a subsequence (still denoted $\varepsilon), u_{\varepsilon}$ converges when $\varepsilon \rightarrow 0$ to some $u$ which is a solution of (1). Identically, $\lambda_{0, \varepsilon}$ and $\lambda_{\gamma, \varepsilon}$ converge to some $\lambda_{0}, \lambda_{\gamma}$ respectively.

Since we have assumed that $\lambda_{0, \varepsilon}$ and $\lambda_{\gamma, \varepsilon}$ behave continuously as $\varepsilon \rightarrow 0$, inequality (29) implies $\lambda_{\gamma} \leq 0 \leq \lambda_{0}$ : we get a contradiction with Lemma 3 .

\subsection{Proof of Theorem 2}

The proof of Theorem 2 follows directly from the following lemma which breaks down the quantity $\lambda_{\gamma}$ into three parts: the stability of the solution, the geometry of the domain (through $\mu_{\gamma}$ from Definition 1), and $\sup f^{\prime}$.

Lemma 4. Let $u$ be a solution of (1) and let $a \geq 1$. Then,

$$
a \lambda_{\gamma} \geq(a-1) \lambda_{0}+\mu_{a \gamma}-\sup f^{\prime} .
$$

If $u$ is not constant, then Theorem 1 implies that $\lambda_{\gamma}<0$, and so Theorem 2 is a direct consequence of the above lemma.

Proof. Fix $b \in(0,1)$ and $\psi \in H^{1}(\Omega)$. By writing $|\nabla \psi|^{2}=b|\nabla \psi|^{2}+(1-b)|\nabla \psi|^{2}$, a straightforward computation gives

$$
\begin{aligned}
& \mathcal{F}_{\gamma}(\psi):=\int_{\Omega}|\nabla \psi|^{2}-\int_{\Omega} f^{\prime}(u)+\int_{\partial \Omega} \gamma \psi^{2} \\
& =b\left(\int_{\Omega}|\nabla \psi|^{2}-\int_{\Omega} f^{\prime}(u) \psi^{2}\right)+(1-b)\left(\int_{\Omega}|\nabla \psi|^{2}+\int_{\partial \Omega} \frac{\gamma}{1-b} \psi^{2}-\int_{\Omega} f^{\prime}(u) \psi^{2}\right) \\
& =b \mathcal{F}_{0}(\psi)+(1-b) \mathcal{G}_{\frac{1}{1-b} \gamma}(\psi)-(1-b) \int_{\Omega} f^{\prime}(u) \psi^{2}
\end{aligned}
$$

with $\mathcal{F}_{\gamma}$ from Definition 3. $\mathcal{F}_{0}$ from Definition 2 and $\mathcal{G}_{\frac{1}{1-b} \gamma}$ from Definition 1. By definition, $\mathcal{F}_{0}(\psi) \geq \lambda_{0} \int_{\Omega} \psi^{2}$ and $\mathcal{G}_{\frac{1}{1-b} \gamma}(\psi) \geq \mu_{\frac{1}{1-b}} \gamma \int_{\Omega}^{1-b} \psi^{2}$. Dividing by $(1-b)$ and setting $a=\frac{1}{1-b}$, we infer

$$
a \mathcal{F}_{\gamma}(\psi) \geq\left((a-1) \lambda_{0}+\mu_{a \gamma}-\sup f^{\prime}\right) \int_{\Omega} \psi^{2} .
$$

We conclude the proof by taking the infimum over all functions $\psi \in H^{1}$ such that $\int_{\Omega} \psi^{2}=1$. 


\section{$5 \quad$ Nonlinear Cacciopoli inequality}

Proof of Theorem 3. Let $u$ be a solution of (8), and set $v_{i}:=\partial_{x_{i}} u$, for all $i \in\{1, \ldots, n\}$. Differentiating (8) with respect to $x_{i}$, we find that $v_{i}$ satisfies

$$
-\Delta v_{i}-\partial_{u} f(x, u) v_{i}-\partial_{x_{i}} f(x, u)=0 \quad \text { in } \Omega .
$$

Multiplying by $v_{i}$ and using the divergence theorem, we find

$$
-\int_{\partial \Omega} v_{i} \partial_{\nu} v_{i}+\int_{\Omega}\left|\nabla v_{i}\right|^{2}=\int_{\Omega} \partial_{u} f(x, u) v_{i}^{2}+\int_{\Omega} \partial_{x_{i}} f(x, u) v_{i} .
$$

Writing $v_{i} \partial_{\nu} v_{i}=\frac{1}{2} \partial_{\nu} v_{i}^{2}$, summing the above equation for $i \in\{1, \ldots, n\}$ and using that $|\nabla u|^{2}=v_{1}^{2}+\cdots+v_{n}^{2}$, we obtain

$$
-\frac{1}{2} \int_{\partial \Omega} \partial_{\nu}\left[|\nabla u|^{2}\right]+\sum_{i=1}^{n} \int_{\Omega}\left|\nabla v_{i}\right|^{2}=\int_{\Omega} \partial_{u} f(x, u)|\nabla u|^{2}+\int_{\Omega} \nabla_{x} f(x, u) \cdot \nabla u .
$$

Let us estimate separately the left and right hand-side of the above expression. To estimate the left hand-side, we use Lemma 1 to deduce that $\frac{1}{2} \partial_{\nu}\left[|\nabla u|^{2}\right] \leq \gamma|\nabla u|^{2}=\gamma \sum_{i=1}^{n} v_{i}^{2}$. Then, recalling the definitions of $\mu_{\gamma}$ and $\mathcal{G}_{\gamma}$ from Definition 1, we have

$$
-\frac{1}{2} \int_{\partial \Omega} \partial_{\nu}\left[|\nabla u|^{2}\right]+\sum_{i=1}^{n} \int_{\Omega}\left|\nabla v_{i}\right|^{2} \geq \sum_{i=1}^{n} \mathcal{G}_{\gamma}\left(v_{i}\right) \geq \mu_{\gamma} \sum_{i=1}^{n} \int_{\Omega} v_{i}^{2}=\mu_{\gamma} \int_{\Omega}|\nabla u|^{2} .
$$

Let us show that the above inequality is strict whenever $u$ is not constant. If there is equality, we have that $\mathcal{G}_{\gamma}\left(v_{i}\right)=\mu_{\gamma} \int_{\Omega} v_{i}^{2}$ for all $i \in\{1, \ldots$,$\} . Then, v_{i}$ minimizes $\mathcal{G}_{\gamma}$ and therefore is a constant multiple of the principal eigenfunction associated to $\mu_{\gamma}$. In particular, $v_{i}$ has a constant strict sign on $\bar{\Omega}$. However, since $\partial_{\nu} u=0$ on $\partial \Omega$, we deduce that $v_{i}$ vanishes on some point of $\partial \Omega$, hence $v_{i} \equiv 0$, and so $u$ is constant. We have thus shown that

$$
-\frac{1}{2} \int_{\partial \Omega} \partial_{\nu}\left[|\nabla u|^{2}\right]+\sum_{i=1}^{n} \int_{\Omega}\left|\nabla v_{i}\right|^{2} \geq \mu_{\gamma} \int_{\Omega}|\nabla u|^{2},
$$

with equality if and only if $u$ is constant.

Now, we compute the right-hand side of (31) using the divergence theorem and that $u$ is a solution of (8):

$$
\begin{aligned}
\int_{\Omega} \partial_{u} f(x, u)|\nabla u|^{2}+\int_{\Omega} \nabla_{x} f(x, u) \cdot \nabla u & =\int_{\Omega} \nabla[f(x, u(x))] \cdot \nabla u \\
& =\int_{\partial \Omega} f(x, u) \partial_{\nu} u d x-\int_{\Omega} f(x, u) \Delta u d x \\
& =\int_{\Omega} f(x, u)^{2} d x .
\end{aligned}
$$

We complete the proof by plugging the above inequality and $(32)$ into 31 . 
Remark 1. With the same method as in Section 4.2 we can use the geometric Poincaré inequality to prove the slightly more precise estimate: for u a nonconstant solution of (8), we have

$$
\mu_{\gamma}\|\nabla u\|^{2}-\int_{\Omega} f(x, u)^{2} d x \leq\|\nabla \mid \nabla u\|^{2}-\left\|\nabla^{2} u\right\|^{2}<0,
$$

where $\|\cdot\|$ denotes the usual $L^{2}$ norm and $\nabla^{2} u$ is the Hessian matrix of $u$.

\section{$6 \quad$ Flatness estimates}

\subsection{Proof of Proposition 3}

Proof of the first statement. Let $\varphi$ be the (unique up to renormalization) eigenfunction associated with $\lambda_{\gamma}[30]$. Denoting $\langle\cdot, \cdot\rangle$ the usual $L^{2}(\Omega)$ scalar product, the mapping $\xi \in \mathbb{R}^{n} \mapsto\langle\nabla u \cdot \xi, \varphi\rangle$ is a continuous linear form which vanishes on a hyperplane $H$. We consider $\left(e_{2}, \ldots, e_{n}\right)$ an orthonormal basis of $H$, that we supplement with a unit vector $e_{1} \in H^{\perp}$ (so that $\left(e_{1}, e_{2}, \ldots, e_{n}\right)$ is an orthonormal basis of $\mathbb{R}^{n}$ ). Then, we set $v_{i}:=\nabla u \cdot e_{i}$.

By construction, $v_{i}$ is orthogonal to $\varphi$ for all $i \in\{2, \ldots, n\}$, hence

$$
\mathcal{F}_{\gamma}\left(v_{i}\right) \geq \begin{cases}\lambda_{\gamma, 2}\left\|v_{i}\right\|^{2} & \text { if } i \in\{2, \ldots, n\} \\ \lambda_{\gamma}\left\|v_{1}\right\|^{2} & \text { if } i=1\end{cases}
$$

From Lemma 2 and that $\|\nabla u\|^{2}=\sum_{i=1}^{n}\left\|v_{i}\right\|^{2}$, we have

$$
0 \geq-\left(\lambda_{\gamma, 2}-\lambda_{\gamma}\right)\left\|v_{1}\right\|^{2}+\lambda_{\gamma, 2}\|\nabla u\|^{2} .
$$

We easily conclude from the above inequality.

Proof of the second statement. As in the previous step, we can choose an orthonormal basis such that, for all $i \in\{2, \ldots, n\}, v_{i}$ is orthogonal to the eigenfunction associated with $\lambda_{0}$. We thus have, for all $a \geq 1$,

$$
\mathcal{F}_{0}\left(v_{i}\right) \geq\left\{\begin{array}{ll}
\lambda_{0,2}\left\|v_{i}\right\|^{2} & \text { if } i \in\{2, \ldots, n\}, \\
\lambda_{0}\left\|v_{1}\right\|^{2} & \text { if } i=1,
\end{array} \quad \text { and } \quad \mathcal{G}_{a \gamma}\left(v_{i}\right) \geq \mu_{a \gamma}\left\|v_{i}\right\|^{2} .\right.
$$

From $(30)$, for all $b \in(0,1)$, we have

$$
0 \geq b \sum_{i=1}^{n} \mathcal{F}_{0}\left(v_{i}\right)+(1-b) \sum_{i=1}^{n} \mathcal{G}_{\frac{1}{1-b} \gamma}\left(v_{i}\right)-(1-b) \int_{\Omega} f^{\prime}(u)|\nabla u|^{2} .
$$

Using (33), we obtain

$$
0 \geq b\left(\lambda_{0}\left\|v_{1}\right\|^{2}+\lambda_{0,2} \sum_{i=2}^{n}\left\|v_{i}\right\|^{2}\right)+(1-b) \mu_{\frac{1}{1-b}} \gamma \sum_{i=1}^{n}\left\|v_{i}\right\|^{2}-(1-b) \sup f^{\prime}\|\nabla u\|^{2} .
$$


Since $\sum_{i=1}^{n}\left\|v_{i}\right\|^{2}=\|\nabla u\|^{2}$, dividing by $(1-b)\|\nabla u\|^{2}$ and setting $a=\frac{1}{1-b}$ gives

$$
0 \geq(a-1)\left(\lambda_{0}\left\|v_{1}\right\|^{2}+\lambda_{0,2}\left(\|\nabla u\|^{2}-\left\|v_{1}\right\|^{2}\right)\right)+\mu_{a \gamma}\|\nabla u\|^{2}-\sup f^{\prime}\|\nabla u\|^{2} .
$$

Dividing by $\|\nabla u\|^{2}$ and rearranging the term gives the result.

Remark 2. An analogous $k$-dimensional estimate can be derived for pattern of Robin-curvature Morse index $k \in\{1, \ldots, n\}$. More precisely, let $u$ be a pattern and define, for all positive integer $k$,

$$
\lambda_{\gamma, k}:=\sup \left\{\inf \left\{\mathcal{F}_{\gamma}(\psi), \psi \in E,\|\psi\|_{L^{2}}=1\right\}, E \subset H^{1}(\Omega), \operatorname{dim} E=k\right\} .
$$

Note that with these notations, we have $\lambda_{\gamma}=\lambda_{\gamma, 1}$. Then, there exists an orthonormal basis $\left(e_{1}, \ldots, e_{n}\right)$ of $\mathbb{R}^{n}$ such that

$$
\sum_{i=1}^{n} \lambda_{\gamma, i}\left\|\nabla u \cdot e_{i}\right\|^{2} \leq 0 .
$$

In particular, if $u$ is of Robin-curvature Morse index $k \in\{1, \ldots, n\}$ (i.e. $\lambda_{\gamma, 1} \leq$ $\left.\cdots \leq \lambda_{\gamma, k-1}<0 \leq \lambda_{\gamma, k}\right)$, then

$$
\frac{\sum_{i=1}^{k}\left\|\nabla u \cdot e_{i}\right\|^{2}}{\|\nabla u\|^{2}} \leq \frac{\lambda_{\gamma, k}}{\lambda_{\gamma, k}-\lambda_{\gamma, 1}},
$$

and $u$ tends to be $k$-dimensional if $\lambda_{\gamma, k} \gg 1$.

Similarily, we can derive an estimate if $u$ is of Morse index $k \in\{1, \ldots, n\}$. Setting, for all positive integer $k$,

$$
\lambda_{0, k}:=\sup \left\{\inf \left\{\mathcal{F}_{0}(\psi), \psi \in E,\|\psi\|_{L^{2}}=1\right\}, E \subset H^{1}(\Omega), \operatorname{dim} E=k\right\},
$$

there exists an orthonormal basis $\left(e_{1}, \ldots, e_{n}\right)$ such that

$$
(a-1)\left(\lambda_{0, k}-\lambda_{0}\right) \frac{\sum_{i=1}^{k}\left\|\nabla u \cdot e_{i}\right\|^{2}}{\|\nabla u\|^{2}} \geq(a-1) \lambda_{0, k}+\mu_{a \gamma}-\sup f^{\prime}, \quad \forall a \geq 1
$$

The same remark holds for Proposition 2, i.e., we can derive a $k$-dimensional estimate involving the $k$-ieth eigenvalue of the Robin-curvature Laplacian. More precisely, define

$\mu_{\gamma, k}:=\sup \left\{\inf \left\{\mathcal{G}_{\gamma}(\psi), \psi \in E,\|\psi\|_{L^{2}}=1\right\}, E \subset H^{1}(\Omega), \operatorname{dim} E=k\right\}, \quad \forall k \in \mathbb{N}$.

Then, there exists an orthonormal basis $\left(e_{1}, \ldots, e_{n}\right)$ of $\mathbb{R}^{n}$ such that $\mathcal{G}\left(v_{i}\right) \geq$ $\mu_{\gamma, i}\left\|v_{i}\right\|^{2}$, and so

$$
0 \geq \sum_{i=1}^{n} \mu_{\gamma, i}\left\|\nabla u \cdot e_{i}\right\|^{2}-\sup f^{\prime}\|\nabla u\|^{2}
$$


In particular, for $u$ a solution of (1) we have that

$$
\left(\mu_{\gamma, k}-\mu_{\gamma, 1}\right) \frac{\sum_{i=1}^{k}\left\|\nabla u \cdot e_{i}\right\|^{2}}{\|\nabla u\|^{2}} \leq \mu_{\gamma, k}-\sup f^{\prime},
$$

and so $u$ tends to be $k$-dimensional if $\mu_{\gamma, k} \gg 1$.

Remark 3. As already mentionned in the proof of Theorem 3, from an integration by part, we have that $\int_{\Omega} f^{\prime}(u)|\nabla u|^{2}=\int_{\Omega} f(u)^{2}$. Hence, the second estimate in Proposition 3 holds if we replace sup $f^{\prime}$ by $\frac{\int_{\Omega} f(u)^{2}}{\|\nabla u\|^{2}}$. Namely, There exists a direction $e \in \mathbb{S}^{n-1}$ such that,

$$
\left(\mu_{\gamma, 2}-\mu_{\gamma}\right) \frac{\|\nabla u \cdot e\|^{2}}{\|\nabla u\|^{2}} \geq \mu_{\gamma, 2}-\frac{\int_{\Omega} f(u)^{2}}{\|\nabla u\|^{2}},
$$

The same remark holds for the estimate in Proposition 2. i.e., there exists a direction $e \in \mathbb{S}^{n-1}$ such that, for all $a \geq 1$,

$$
(a-1)\left(\lambda_{0,2}-\lambda_{0}\right) \frac{\|\nabla u \cdot e\|^{2}}{\|\nabla u\|^{2}} \geq(a-1) \lambda_{0,2}+\mu_{a \gamma}-\frac{\int_{\Omega} f(u)^{2}}{\|\nabla u\|^{2}} .
$$

\subsection{Proof of Proposition 2}

As in the proof of Proposition 2 we can choose an orthonormal basis such that, for all $i \in\{2, \ldots, n\}, v_{i}$ is orthogonal to the eigenfunction associated with $\mu_{\gamma}$. Hence,

$$
\mathcal{G}_{\gamma}\left(v_{i}\right) \geq \begin{cases}\mu_{\gamma, 2}\left\|v_{i}\right\|^{2} & \text { if } i \in\{2, \ldots, n\} \\ \mu_{\gamma}\left\|v_{1}\right\|^{2} & \text { if } i=1 .\end{cases}
$$

From 30 with $b=0$ and $\psi=v_{i}$, we have

$$
\mathcal{F}_{\gamma}\left(v_{i}\right)=\mathcal{G}_{\gamma}\left(v_{i}\right)-\int_{\Omega} f^{\prime}(u) v_{i}^{2} .
$$

Summing the above equality for $i=1, \ldots, n$ and using Lemma 2 we infer

$$
0 \geq \sum_{i=1}^{n} \mathcal{G}_{\gamma}\left(v_{i}\right)-\sum_{i=1}^{n} \int_{\Omega} f^{\prime}(u) v_{i}^{2} .
$$

Using (34) and that $|\nabla u|^{2}=v_{1}^{2}+\cdots+v_{n}^{2}$, we obtain

$$
0 \geq \mu_{\gamma}\left\|v_{1}\right\|^{2}+\mu_{\gamma, 2}\left(\|\nabla u\|^{2}-\left\|v_{1}\right\|^{2}\right)-\sup f^{\prime}\|\nabla u\|^{2} .
$$

We easily conclude from the above inequality.

Remark 4. Using the same method as in the proof of Proposition 3, we can show that, for any $a \geq 1$, there exists a direction $e \in \mathbb{S}^{n-1}$ such that

$$
\left(\mu_{a \gamma, 2}-\mu_{a \gamma}\right) \frac{\|\nabla u \cdot e\|^{2}}{\|\nabla u\|^{2}} \geq(a-1) \lambda_{0}+\mu_{a \gamma, 2}-\sup f^{\prime} .
$$




\section{Unbounded domains}

\subsection{Proof of Theorem 4. First statement}

First, note that Lemma 1 holds in unbounded domains. However, it is not the case for Lemma 2 because $v_{i}$ may not belong to $H^{1}(\Omega)$ and thus the computation of $\mathcal{F}\left(v_{i}\right)$ is not licit. Let us consider the cut-off function defined in $\left.\sqrt{19}\right)$. We begin with a lemma which adapts Lemma 2 to unbounded domains.

Lemma 5. Let $u$ be a solution of 13 , and set $v_{i}:=\partial_{x_{i}} u$, for all $i \in\{1, \ldots, n\}$. We have that

$$
\lambda_{\gamma}^{R} \int_{\Omega} \chi_{R}^{2}|\nabla u|^{2} \leq \sum_{i=1}^{n} \mathcal{F}_{\gamma}\left(\chi_{R} v_{i}\right) \leq \int_{\Omega}\left|\nabla \chi_{R}\right|^{2}|\nabla u|^{2}, \quad \forall R>0
$$

with $\mathcal{F}_{\gamma}$ from Definition 3 .

Proof. The first inequality is directly deduced from $|\nabla u|^{2}=v_{1}^{2}+\cdots+v_{n}^{2}$ and $\lambda_{\gamma}\left\|\chi_{R} v_{i}\right\|^{2} \leq \mathcal{F}_{\gamma}\left(\chi_{R} v_{i}\right)$. Let us focus on the second inequality. Differentiating (13) with respect to $x_{i}$, we find that $v_{i}$ satisfies the linearized equation

$$
-\Delta v_{i}-f^{\prime}(u) v_{i}=0 \quad \text { in } \Omega
$$

Multiplying by $\chi_{R}^{2} v_{i}$, integrating on $\Omega$ and using the divergence theorem, we find

$$
-\int_{\partial \Omega} \chi_{R}^{2} v_{i} \partial_{\nu} v_{i}+\int_{\Omega} \nabla\left[\chi_{R}^{2} v_{i}\right] \cdot \nabla v_{i}-\int_{\Omega} f^{\prime}(u) \chi_{R}^{2} v_{i}^{2}=0 .
$$

Using $\left|\nabla\left[\chi_{R} v_{i}\right]\right|^{2}=\nabla\left[\chi_{R}^{2} v_{i}\right] \cdot \nabla v_{i}+\left|\nabla \chi_{R}\right|^{2} v_{i}^{2}$ and $v_{i} \partial_{\nu} v_{i}=\frac{1}{2} \partial_{\nu} v_{i}^{2}$, we derive

$$
\int_{\Omega}\left|\nabla\left[\chi_{R} v_{i}\right]\right|^{2}-\int_{\Omega} f^{\prime}(u) \chi_{R}^{2} v_{i}^{2}=\int_{\partial \Omega} \frac{1}{2} \partial_{\nu} v_{i}^{2} \chi_{R}^{2}+\int_{\Omega}\left|\nabla \chi_{R}\right|^{2} v_{i}^{2},
$$

and so

$$
\mathcal{F}_{\gamma}\left(\chi_{R} v_{i}\right)=\int_{\partial \Omega}\left(\frac{1}{2} \partial_{\nu} v_{i}^{2} \chi_{R}^{2}+\gamma v_{i}^{2}\right)+\int_{\Omega}\left|\nabla \chi_{R}\right|^{2} v_{i}^{2} .
$$

Summing the above expression for $i \in\{1, \ldots, n\}$ and using that $|\nabla u|^{2}=v_{1}^{2}+$ $\cdots+v_{n}^{2}$ gives

$$
\sum_{i=1}^{n} \mathcal{F}_{\gamma}\left(\chi_{R} v_{i}\right)=\int_{\partial \Omega} \chi_{R}^{2}\left(\frac{1}{2} \partial_{\nu}|\nabla u|^{2}+\gamma|\nabla u|^{2}\right)+\int_{\Omega}\left|\nabla \chi_{R}\right|^{2}|\nabla u|^{2} .
$$

The first term in the right-hand side of the above identity is nonpositive from Lemma 1, which completes the proof.

We are now ready to prove the first assertion of Theorem 4. 
Proof of Theorem 4 first statement. Assume that (17) holds, and assume by contradiction that $u$ is not constant. On the one hand, from the definition of $\lambda_{\gamma}^{R}$ in $(14)$, we have

$$
\sum_{i=1}^{n} \mathcal{F}_{\gamma}\left(\chi_{R} v_{i}\right) \geq \lambda_{\gamma}^{R} \int_{\Omega} \chi_{R}^{2}|\nabla u|^{2} \geq \lambda_{\gamma}^{R} \int_{\Omega_{R}}|\nabla u|^{2} .
$$

On the other hand, we have that

$$
\int_{\Omega_{R}}\left|\nabla \chi_{R}\right|^{2}|\nabla u|^{2} \leq \frac{4}{R^{2}} \int_{\Omega_{2 R}}|\nabla u|^{2} .
$$

Using the two above inequalities in 35 , we deduce

$$
\frac{1}{4} R^{2} \lambda_{\gamma}^{R} \leq \frac{\int_{\Omega_{2 R}}|\nabla u|^{2}}{\int_{\Omega_{R}}|\nabla u|^{2}}
$$

Notice that, since $u$ is not constant, the strong maximum principle implies that $u$ cannot be constant on any open set, and therefore $\int_{\Omega_{R}}|\nabla u|^{2}>0$. Iterating the above inequality, we find, for $j=1,2, \ldots$,

$$
\left(\frac{R^{2} \lambda_{\gamma}^{R}}{4}\right)^{j} \leq \frac{\int_{\Omega_{2^{j} R}}|\nabla u|^{2}}{\int_{\Omega_{R}}|\nabla u|^{2}}
$$

Since $\nabla u$ is bounded and not identically zero, there exists a constant $K$ such that

$$
\frac{\int_{\Omega_{2^{j} R}}|\nabla u|^{2}}{\int_{\Omega_{R}}|\nabla u|^{2}} \leq K\left(2^{j} R\right)^{n} .
$$

Therefore,

$$
\left(\frac{R^{2} \lambda_{\gamma}^{R}}{2^{n+2}}\right)^{j} \leq K R^{n}
$$

From (17), we can choose $R>0$ such that $R^{2} \lambda_{\gamma}^{R}>2^{n+2}$; then, we reach a contradiction letting $j \rightarrow+\infty$.

\subsection{Proof of Theorem 4. Second and third statements.}

Even if the domain is unbounded, $\lambda_{\gamma}$ defined through 15 is associated with an eigenfunction $\varphi$ which is positive on $\bar{\Omega}$ and satisfies

$$
\begin{cases}-\Delta \varphi-f^{\prime}(u) \varphi=\lambda_{\gamma} \varphi & \text { in } \Omega \\ \partial_{\nu} \varphi+\gamma \varphi=0 & \text { on } \partial \Omega .\end{cases}
$$

The existence of $\varphi$ is proved by Rossi in [58]. The function $\varphi$ is often refered to as a generalized principal eigenfunction, because $\varphi$ might not belong to $H^{1}(\Omega)$.

If the domain is bounded, it is classical that $\lambda_{\gamma}$ is a simple eigenvalue of (37). This property also holds in unbounded domains satisfying $[16$, as a consequence of the following lemma which is a refinement of [11, Theorem 1.7]. 
Lemma 6. Assume that $\Omega \subset \mathbb{R}^{n}$ satisfies (16) and let $u$ be a Robin-curvaturestable solution of (13). If $v$ is smooth, bounded and satisfies

$$
v\left(-\Delta v-f^{\prime}(u) v\right) \leq 0, \quad \text { in } \Omega,
$$

and

$$
v\left(\partial_{\nu} v+\gamma v\right) \leq 0, \quad \text { on } \partial \Omega,
$$

then $v \equiv C \varphi$ for some constant $C$, where $\varphi$ is a principal eigenfunction associated with $\lambda_{\gamma}$.

Proof. Let us set $\sigma=\frac{v}{\varphi}$ and show that $\sigma$ is constant. Since $v$ satisfies (38)-(39), a straightforward computation shows that $\sigma$ satisfies

$$
\sigma \varphi\left(\varphi \Delta \sigma+2 \nabla \varphi \cdot \nabla \sigma+\sigma\left(\Delta \varphi+f^{\prime}(u) \varphi\right)\right) \geq 0 .
$$

Using that $\varphi$ satisfies (37) and that $\lambda_{\gamma} \geq 0$, we have

$$
\varphi \sigma^{2}\left(\Delta \varphi+f^{\prime}(u) \varphi\right) \leq-\lambda_{\gamma} \varphi^{2} \sigma^{2} \leq 0
$$

therefore,

$$
\sigma \varphi(\varphi \Delta \sigma+2 \nabla \varphi \cdot \nabla \sigma) \geq 0
$$

which can be rewritten as

$$
\sigma \nabla \cdot\left(\varphi^{2} \nabla \sigma\right) \geq 0
$$

Multiplying by $\chi_{R}^{2}$ (defined in $(19)$ ), integrating on $\Omega$ and using the divergence theorem, we find

$$
\begin{aligned}
0 & \leq \int_{\partial \Omega} \chi_{R}^{2} \sigma \varphi^{2} \partial_{\nu} \sigma-\int_{\Omega} \varphi^{2} \nabla\left(\chi_{R}^{2} \sigma\right) \cdot \nabla \sigma \\
& =\int_{\partial \Omega} \chi_{R}^{2} \sigma \varphi^{2} \partial_{\nu} \sigma-\int_{\Omega} \varphi^{2} \chi_{R}^{2}|\nabla \sigma|^{2}-2 \int_{\Omega} \varphi^{2} \chi_{R} \sigma \nabla \chi_{R} \cdot \nabla \sigma .
\end{aligned}
$$

Since $\partial_{\nu} \varphi=-\gamma \varphi$, the boundary term reads

$$
\int_{\partial \Omega} \chi_{R}^{2} \sigma \varphi^{2} \partial_{\nu} \sigma=\int_{\partial \Omega} \chi_{R}^{2} v\left[\partial_{\nu} v-\gamma v\right]
$$

which is nonpositive from (38). From Cauchy-Schwarz inequality, we deduce

$$
\int_{\Omega} \chi_{R}^{2} \varphi^{2}|\nabla \sigma|^{2} \leq 2 \sqrt{\int_{\Omega_{2 R} \backslash \Omega_{R}} \chi_{R}^{2} \varphi^{2}|\nabla \sigma|^{2}} \sqrt{\int_{\Omega} v^{2}\left|\nabla \chi_{R}\right|^{2}},
$$

where $\Omega_{R}=\Omega \cap\{|x| \leq R\}$.

The assumption on the growth of the domain at infinity (16) implies

$$
\int_{\Omega} v^{2}\left|\nabla \chi_{R}\right|^{2} \text { is bounded, uniformly in } R \geq 1 .
$$


From 40, we deduce that $\int_{\Omega} \chi_{R}^{2} \varphi^{2}|\nabla \sigma|^{2}$ is uniformly bounded. Hence,

$$
\lim _{R \rightarrow+\infty}\left[\int_{\Omega_{2 R} \backslash \Omega_{R}} \chi_{R}^{2} \varphi^{2}|\nabla \sigma|^{2}\right]=0 .
$$

At the limit as $R \rightarrow+\infty$ in 40 , we find $\int_{\Omega} \varphi^{2}|\nabla \sigma|^{2} \leq 0$. Hence $\nabla \sigma=0$, which achieves the proof.

The cornerstone of the proof is that $\sigma \nabla \cdot\left(\varphi^{2} \nabla \sigma\right) \geq 0$ implies $\nabla \sigma=0$, where $\sigma:=\frac{v}{\varphi}$. The litterature refers to this property as a Liouville property. Originally introduced in [1], it has been extensively used and discussed $9,38,53,67$. For more details, we refer to our previour paper [54, Section 3.2]. Note that this is the only step where $(16)$ is needed. In our context, this condition is essentially optimal, as recently proved by Villegas 67 .

We are now ready to complete the proof of Theorem 4

Proof of Theorem 4, second and third statements. Let $\varphi$ be a principal eigenfunction associated with $\lambda_{\gamma}$. Let us first prove that $v_{i}:=\partial_{x_{i}} u$ is a constant multiple of $\varphi$, for all $i=1, \ldots, n$. First, differentiating (13), we find that $v_{i}$ satisfies the first equation in (38). Then, we show that all the $v_{i}$ satisfy the boundary condition in (39). Notice that if $v_{i}$ indeed satisfies (39), then Lemma 6 implies that $v_{i}$ is a constant multiple of $\varphi$ and therefore $\partial_{\nu} v_{i}+\gamma v_{i}=0$. Hence, we know in general that $v_{i}\left(\partial_{\nu} v_{i}+\gamma v_{i}\right) \geq 0$. However, Lemma 1 implies

$$
\sum_{i=1}^{n} v_{i}\left(\partial_{\nu} v_{i}+\gamma v_{i}\right) \leq 0
$$

We deduce that $v_{i}\left(\partial_{\nu} v_{i}+\gamma v_{i}\right)=0$. Applying Lemma 6 , we deduce that $v_{i}$ is a constant multiple of $\varphi$.

The previous step implies that there exists a constant vector $\mathbf{C} \in \mathbb{R}^{n}$ such that $\nabla u \equiv \mathbf{C} \varphi$. Hence, $u$ is a planar function. If the domain is not a straight cylinder, there exists $x_{0} \in \partial \Omega$ such that $\nu\left(x_{0}\right) \cdot \mathbf{C} \neq 0$. Then, the Neumann boundary condition in 130 implies that $\nabla u\left(x_{0}\right)=0$. Since $\varphi>0$ on $\bar{\Omega}$, we deduce that $\mathbf{C}=0$, hence $u$ is constant. It completes the proof of the second statement of Theorem 4

Assume that $\Omega$ is a straight cylinder of the form $\mathbb{R} \times \omega, \omega \subset \mathbb{R}^{n-1}$, and that $u$ only depends on $x_{1}$. Since $u^{\prime}$ is a constant multiple of $\varphi>0, u$ is monotonic. Assume in addition that $u$ is bounded. Then, $u$ has limits $u( \pm \infty)=z^{ \pm}$. If $z^{+}=$ $z^{-}$, then $u$ is constant. Let us assume $z^{-} \neq z^{+}$, and fix $M>0$. Multiplying $-u^{\prime \prime}=f(u)$ by $u^{\prime}$ and integrating on $x_{1} \in[-M, M]$ gives

$$
\frac{1}{2}\left(u^{\prime}(-M)^{2}-u^{\prime}(M)^{2}\right)=\int_{u(-M)}^{u(M)} f .
$$

Since $u^{\prime}( \pm \infty)=0$ (indeed, $u^{\prime}$ is integrable and $u^{\prime \prime}$ is bounded), when $M$ goes to $+\infty$ we obtain $\int_{z^{-}}^{z^{+}} f=0$. The proof of the third statement of Theorem 4 is thereby complete. 


\subsection{Proof of Corollary 3}

Let $u$ be a solution of 13 . Notice that Lemma 4 remains true when the domain is unbounded, and so

$$
a \lambda_{\gamma} \geq(a-1) \lambda_{0}+\mu_{a \gamma}-\sup f^{\prime}, \quad \forall a \geq 1 .
$$

If $u$ is not constant, then Theorem 4 implies that $\lambda_{\gamma} \leq 0$. Then, the proof of Theorem 2 directly follows from the above inequality.

\subsection{Proof of Theorem 5}

Let $u$ be a solution of 20$)$ and set $v_{i}:=\partial_{x_{i}} u$, for all $i \in\{1, \ldots, n\}$. Differentiating (20) with respect to $x_{i}$, we find that $v_{i}$ satisfies

$$
-\Delta v_{i}-\partial_{u} f(x, u) v_{i}-\partial_{x_{i}} f(x, u)=0 \quad \text { in } \Omega .
$$

Multiplying by $\chi_{R}^{2} v_{i}$ (we recall that $\chi_{R}$ is defined in (19) ) and using the divergence theorem, we find

$$
-\int_{\partial \Omega} \chi_{R}^{2} v_{i} \partial_{\nu} v_{i}+\int_{\Omega} \nabla\left[\chi_{R}^{2} v_{i}\right] \cdot \nabla v_{i}=\int_{\Omega} \partial_{u} f(x, u) v_{i}^{2} \chi_{R}^{2}+\int_{\Omega} \partial_{x_{i}} f(x, u) v_{i} \chi_{R}^{2}
$$

Summing the above equation for $i \in\{1, \ldots, n\}$, and using that $\left|\nabla\left[\chi_{R} v_{i}\right]\right|^{2}=$ $\nabla\left[\chi_{R}^{2} v_{i}\right] \cdot \nabla v_{i}+\left|\nabla \chi_{R}\right|^{2} v_{i}^{2}$, that $v_{i} \partial_{\nu} v_{i}=\frac{1}{2} \partial_{\nu} v_{i}^{2}$, and that $|\nabla u|^{2}=v_{1}^{2}+\cdots+v_{n}^{2}$, we obtain

$$
\begin{aligned}
& -\frac{1}{2} \int_{\partial \Omega} \chi_{R}^{2} \partial_{\nu}\left[|\nabla u|^{2}\right]+\sum_{i=1}^{n} \int_{\Omega}\left|\chi_{R}^{2} \nabla v\right|^{2} \\
& \quad=\int_{\Omega}\left|\nabla \chi_{R}\right|^{2}|\nabla u|^{2} \int_{\Omega} \chi_{R}^{2} \partial_{u} f(x, u)|\nabla u|^{2}+\int_{\Omega} \chi_{R}^{2} \nabla_{x} f(x, u) \cdot \nabla u .
\end{aligned}
$$

Let us first estimate the left hand-side of the above expression. We use Lemma 1 to deduce that $\frac{1}{2} \partial_{\nu}\left[|\nabla u|^{2}\right] \leq \gamma|\nabla u|^{2}=\gamma \sum_{i=1}^{n} v_{i}^{2}$. Therefore, recalling the definitions of $\mu_{\gamma}$ and $\mathcal{G}_{\gamma}$ from Definition 1 , we have

$$
\begin{aligned}
-\frac{1}{2} \int_{\partial \Omega} \chi_{R}^{2} \partial_{\nu}\left[|\nabla u|^{2}\right]+\sum_{i=1}^{n} \int_{\Omega}\left|\chi_{R}^{2} \nabla v_{i}\right|^{2} \geq \sum_{i=1}^{n} \mathcal{G}_{\gamma}\left(\chi_{R}^{2} v_{i}\right) & \geq \mu_{\gamma}^{R} \sum_{i=1}^{n} \int_{\Omega} \chi_{R}^{2} v_{i}^{2} \\
& =\mu_{\gamma}^{R} \int_{\Omega} \chi_{R}^{2}|\nabla u|^{2} .
\end{aligned}
$$

Now, we estimate the right-hand side of 411) using the divergence theorem 
and that $u$ is a solution of 20$]$ :

$$
\begin{aligned}
& \int_{\Omega}\left|\nabla \chi_{R}\right|^{2}|\nabla u|^{2}+\int_{\Omega} \chi_{R}^{2} \partial_{u} f(x, u)|\nabla u|^{2} d x+\int_{\Omega} \chi_{R}^{2} \nabla_{x} f(x, u) \cdot \nabla u d x \\
& =\int_{\Omega}\left|\nabla \chi_{R}\right|^{2}|\nabla u|^{2}+\int_{\Omega} \chi_{R}^{2} \nabla[f(x, u(x))] \cdot \nabla u d x \\
& =\int_{\Omega}\left|\nabla \chi_{R}\right|^{2}|\nabla u|^{2}+\int_{\partial \Omega} \chi_{R}^{2} f(x, u(x)) \partial_{\nu} u-\int_{\Omega} f(x, u(x)) \nabla \cdot\left[\chi_{R}^{2} \nabla u\right] d x \\
& =\int_{\Omega}\left|\nabla \chi_{R}\right|^{2}|\nabla u|^{2}+\int_{\Omega} \chi_{R}^{2} f(x, u(x))^{2} d x-2 \int_{\Omega} \chi_{R} f(x, u) \nabla \chi_{R} \cdot \nabla u, \\
& \leq \int_{\Omega}\left(\left|\nabla \chi_{R}\right||\nabla u|+f(x, u) \chi_{R}\right)^{2} d x \text {. }
\end{aligned}
$$

We complete the proof of Theorem 5 by plugging the above inequality and (42) into 41.

\subsection{Proof of Corollary 4}

First, notice that, if $u$ is not constant, the strong maximum principle implies that $u$ is not constant on any open set, and therefore $\int_{\Omega_{R}}|\nabla u|^{2}>0$ for all $R>0$. Hence the expression in Corollary 4 is well defined.

From (21), we have that

$$
\mu_{\gamma}^{R} \int_{\Omega} \chi_{R}^{2}|\nabla u|^{2} \leq 2 \int_{\Omega}\left|\nabla \chi_{R}\right|^{2}|\nabla u|^{2}+2 \int_{\Omega} \chi_{R}^{2} f(x, u)^{2} d x .
$$

To prove Corollary 4 it amounts to show that

$$
\liminf _{R \rightarrow+\infty} \frac{\int_{\Omega}\left|\nabla \chi_{R}\right|^{2}|\nabla u|^{2}}{\int_{\Omega} \chi_{R}^{2}|\nabla u|^{2}}=0 .
$$

By contradiction, assume that the above lim inf is strictly positive. We deduce that there exists $\alpha>0$ such that for all $R>0$ we have

$$
\frac{\int_{\Omega_{2 R}}|\nabla u|^{2}}{\int_{\Omega_{R}}|\nabla u|^{2}} \geq \alpha R^{2}
$$

Here, we have used the definition of $\chi_{R}$ in $(19)$, in particular, that $\left|\nabla \chi_{R}\right|^{2}=$ $O\left(R^{-2}\right)$.

This inequality is similar to 36 in the proof of Theorem 4 . However, we have shown there that (36) leads to a contradiction. The proof is thereby achieved.

\section{References}

[1] N. D. Alikakos and P. W. Bates. On the singular limit in a phase field model of phase transitions. Annales de l'Institut Henri Poincaré (C), 5(2), 1988. 
[2] B. Andrews and J. Clutterbuck. Proof of the fundamental gap conjecture. Journal of the Americal Mathematical Society, 24(3), 2010.

[3] J. M. Arrieta, A. N. Carvalho, and G. Lozada-Cruz. Dynamics in dumbbell domains I. Continuity of the set of equilibria. Journal of Differential Equations, 231:551-597, 2006.

[4] J. M. Arrieta, A. N. Carvalho, and G. Lozada-Cruz. Dynamics in dumbbell domains II. The limiting problem. Journal of Differential Equations, 247(247):174-202, 2009.

[5] J. M. Arrieta, A. N. Carvalho, and G. Lozada-Cruz. Dynamics in dumbbell domains III. Continuity of attractors. Journal of Differential Equations, $247(247): 225-259,2009$.

[6] C. Bandle, P. Mastrolia, D. Monticelli, and F. Punzo. On the stability of solutions of semilinear elliptic equations with Robin boundary conditions on Riemannian manifolds. SIAM Journal on Mathematical Analysis, 48(1), 2016.

[7] C. Bandle, F. Punzo, and A. Tesei. Existence and nonexistence of patterns on Riemannian manifolds. Journal of Mathematical Analysis and Applications, 387(1):33-47, 2012.

[8] M. Bareket and B. Rulf. An eigenvalue problem related to sound propagation in elastic tubes. Journal of Sound and Vibration, 38(4):437-449, 1975 .

[9] M. T. Barlow, R. F. Bass, and C. Gui. The Liouville property and a conjecture of De Giorgi. Communications on Pure and Applied Mathematics, 53(8):1007-1038, 2000.

[10] H. Berestycki, J. Bouhours, and G. Chapuisat. Front blocking and propagation in cylinders with varying cross section. Calculus of Variations and Partial Differential Equations, 55(3):44, 2016.

[11] H. Berestycki, L. Caffarelli, and L. Nirenberg. Further qualitative properties for elliptic equations in unbounded domains. Annali della Scuola Normale Superiore di Pisa, Classe di Scienze 4e série, 25(1-2):69-94, 1997.

[12] H. Berestycki, F. Hamel, and H. Matano. Bistable travelling waves around an obstacle. Communications in Pure and Applied Mathematics, 62(6):729$788,2009$.

[13] H. Berestycki and L. Nirenberg. Travelling fronts in cylinders. Annales de l'Institut Henri Poincaré (C) Analyse non linéaire, 9(5):497-572, 1992.

[14] J. Brasseur and J. Coville. A Counterexample to the Liouville Property of some nonlocal problems. ArXiv Preprint, 2018. 
[15] J. Brasseur, J. Coville, F. Hamel, and E. Valdinoci. Liouville type results for a nonlocal obstacle problem. Proceedings of the London Mathematical Society, feb 2019.

[16] K. J. Brown and P. Hess. Stability and Uniqueness of Positive Solutions for a Semi-linear Elliptic Boundary Value Problem. Differential and Integral Equations, 3(2):201-207, 1990.

[17] X. Cabré, A. Figalli, X. Ros-Oton, and J. Serra. Stable solutions to semilinear elliptic equations are smooth up to dimension 9. to appear, 2019.

[18] L. A. Caffarelli and A. Córdoba. Phase transitions: Uniform regularity of the intermediate layers. Journal fur die reine und angewandte Mathematik (Crelles Journal), 593:209-235, 2006.

[19] J. W. Cahn and J. E. Hilliard. Free energy of a nonuniform system. I. Interfacial free energy. The Journal of Chemical Physics, 28(2):258-267, feb 1958.

[20] R. G. Casten and C. J. Holland. Instability results for reaction diffusion equations with Neumann boundary conditions. Journal of Differential Equations, 27(2):266-273, 1978.

[21] S. Chanillo and X. Cabré. Stable solutions of semilinear elliptic problems in convex domains. Selecta Mathematica, New Series, 4(1):1-9, 1998.

[22] M.-F. Chen and F.-Y. Wang. Estimation of Spectral Gap for Elliptic Operators. Transaction of the American Mathematical Society, 349(397):12391267, 1997.

[23] M.-F. Chen and F.-Y. Wang. General formula for lower bound of the first eigenvalue on Riemannian manifolds. Science in China (A), 40(4), 1997.

[24] G. Ciraolo, R. Corso, and A. Roncoroni. Classification and non-existence results for weak solutions to quasilinear elliptic equations with Neumann or Robin boundary conditions. arXiv preprint, pages 1-19, 2020.

[25] N. Cònsul and J. Solà-Morales. Stability of local minima and stable nonconstant equilibria. Journal of Differential Equations, 157(1):61-81, sep 1999 .

[26] E. N. Dancer. Stable and Finite Morse Index solutions on $\mathrm{R}^{\wedge} \mathrm{n}$ or on bounded domains with small diffusion. Transactions of the American Mathematical Society, 357(304):1225-1243, 2004.

[27] E. N. Dancer and D. Daners. Domain Perturbation for Elliptic Equations Subject to Robin Boundary Conditions. Journal of Differential Equations, $138,1997$. 
[28] D. Daners. Local singular variation of domain for semilinear elliptic equations. In Topics in Nonlinear Analysis, pages 117-141. Birkhäuser Basel, Basel, 1999.

[29] D. Daners. Domain Perturbation for Linear and Semi-Linear Boundary Value Problems. Handbook of Differential Equations: Stationary Partial Differential Equations, 6(1):1-81, 2008.

[30] D. Daners. Principal Eigenvalues for Generalised Indefinite Robin Problems. Potential Anal, 38:1047-1069, 2013.

[31] S. Dipierro, A. Pinamonti, and E. Valdinoci. Classification of stable solutions for boundary value problems with nonlinear boundary conditions on Riemannian manifolds with nonnegative Ricci curvature. Advances in Nonlinear Analysis, 8(1):1035-1042, jun 2018.

[32] S. Dipierro, A. Pinamonti, and E. Valdinoci. Rigidity Results for Elliptic Boundary Value Problems: Stable Solutions for Quasilinear Equations with Neumann or Robin Boundary Conditions. International Mathematics Research Notices, pages 1-16, 2018.

[33] S. Dipierro, N. Soave, and E. Valdinoci. On stable solutions of boundary Reaction-Diffusion equations and applications to nonlocal problams with Neumann data. Indiana University Mathematics Journal, 67(1), 2016.

[34] A. S. Do Nascimento and M. S. Sônego. Patterns on surfaces of revolution in a diffusion problem with variable diffusivity. Electronic Journal of Differential Equations, 2014(238):1-13, 2014.

[35] L. Dupaigne. Stable Solutions of Elliptic Partial Differential Equations. Monographs \& Surveys in Pure \& Applied Math. Chapman and Hall/CRC, mar 2011.

[36] A. Farina, L. Mari, and E. Valdinoci. Splitting Theorems, Symmetry Results and Overdetermined Problems for Riemannian Manifolds. Communications in Partial Differential Equations, 38(10):1818-1862, 2013.

[37] A. Farina, Y. Sire, and E. Valdinoci. Stable Solutions of Elliptic Equations on Riemannian Manifolds. Journal of Geometric Analysis, 23:1158-1172, 2013.

[38] F. Gazzola. The sharp exponent for a Liouville-type theorem for an elliptic inequality. Rendiconti dell'Istituto di Matematica dell'Università di Trieste, 34(1-2):99-102, 2003.

[39] D. Gilbarg and N. S. Trudinger. Elliptic Partial Differential Equations of Second Order, volume 1542. Springer-Verlag, Berlin, Heidelberg, 2001.

[40] M. Gokieli and N. Varchon. Stability and instability of equilibria on singular domains. In Nonlocal and Abstract Parabolic Equations and their Applications, Bedlewo, Poland, 2009. Banach Center Publications 86. 
[41] A. C. Gonçalves and A. S. Nascimento. Instability of elliptic equations on compact Riemannian manifolds with non-negative Ricci Curvature. Electronic Journal of Differential Equations, 2010(67):1-18, 2010.

[42] J. K. Hale. Eigenvalues and perturbed domains. 10 Mathematical Essays on Approximation in Analysis and Topology, pages 95-123, 2005.

[43] J. K. Hale and J. Vegas. A nonlinear parabolic equation with varying domain. Archive for Rational Mechanics and Analysis, 86(2):99-123, 1984.

[44] D. Henry. Perturbation of the Boundary in Boundary-Value Problems of Partial Differential Equations. Cambridge University Press, Cambridge, 2005.

[45] S. Jimbo. On a semilinear diffusion equation on a Riemannian manifold and its stable equilibrium solutions. Proceedings of the Japan Academy, Series A, Mathematical Sciences, 60(10):349-352, 1984.

[46] S. Jimbo and Y. Morita. Stability of nonconstant steady-state solutions to a Ginzburg-Landau equation in higher space dimensions. Nonlinear Analysis: Theory, Methods \& Applications, 22(6):753-770, mar 1994.

[47] R. V. Kohn and P. Sternberg. Local minimisers and singular perturbations. Proceedings of the Royal Society of Edinburgh, A, 111(1-2):69-84, 1989.

[48] H. Kovařík. On the lowest eigenvalue of Laplace operator with mixed boundary conditions. Journal of Geometric Analysis, 24(3):1509-1525, 2012.

[49] M. Levitin and L. Parnovski. On the principal eigenvalue of a Robin problem with a large parameter. Mathematische Nachrichten, 281(2):272-281, 2008.

[50] H. Matano. Asymptotic behavior and stability of solutions of semilinear diffusion equations. Publications of the Research Institute for Mathematical Sciences, 15(2):401-454, 1979.

[51] L. Modica. Convergence to minimal surfaces problem and global solutions of Delta $\mathrm{u}=2\left(\mathrm{u}^{\wedge} 3-\mathrm{u}\right)$. In Proceedings of the International Meeting on Recent Methods in Nonlinear Analysis (Rome, 1978), pages 223-244, Bologna, 1979. Pitagora.

[52] L. Modica and S. Mortola. Un esempio di Gamma-convergenza. Bollettino della Unione Matematica Italiana B, 14:285-299, 1977.

[53] L. Moschini. New Liouville theorems for linear second order degenerate elliptic equations in divergence form. Annales de l'Institut Henri Poincare (C) Non Linear Analysis, 22(1):11-23, 2005. 
[54] S. Nordmann. Symmetry properties of stable solutions of semilinear elliptic equations in unbounded domains. to appear in Calculus of Variations and Partial Differential Equations, 2020.

[55] F. Pacard and J. Wei. Stable solutions of the Allen-Cahn equation in dimension 8 and minimal cones. Journal of Functional Analysis, 264(5):11311167, mar 2013.

[56] F. Punzo. The existence of patterns on surfaces of revolution without boundary. Nonlinear Analysis: Theory, Methods \&3 Applications, 77:94102, jan 2013.

[57] J. Rohleder. Strict inequality of Robin eigenvalues for elliptic differential operators on Lipschitz domains. ArXiv preprint, 2014.

[58] L. Rossi. Stability analysis for semilinear parabolic problems in general unbounded domains. arXiv preprint, 2020.

[59] J. Rubinstein and G. Wolansky. Instability results for reaction diffusion equations over surfaces of revolutions. Journal of Mathematical Analysis and Applications, 187(2):485-489, oct 1994.

[60] O. Savin. Phase transitions, minimal surfaces and a conjecture of De Giorgi. Annals of Mathematics, 169:41-78, 2009.

[61] A. Savo. Optimal eigenvalue estimates for the Robin Laplacian on Riemannian manifolds. ArXiv preprint, 2019.

[62] M. S. Sônego. Patterns in a balanced bistable equation with heterogeneous environments on surfaces of revolution. Differential Equations $\mathcal{E}$ Applications, 8(4):521-533, 2016.

[63] M. S. Sônego. Existence of radially symmetric patterns for a diffusion problem with variable diffusivity. Electronic Journal of Qualitative Theory of Differential Equations, 64:1-10, 2017.

[64] P. Sternberg and K. Zumbrun. A Poincaré inequality with applications to volume-constrained area-minimizing surfaces. J. Reine Angew. Math., 503:63-85, 1998.

[65] P. Sternberg and K. Zumbrun. Connectivity of phase boundaries in strictly convex domains. Archive for Rational Mechanics and Analysis, 141:375400, 1998.

[66] K. Umezu. On eigenvalue problems with Robin type boundary conditions having indefinite coefficients. Applicable Analysis, 85(11):1313-1325, 2006.

[67] S. Villegas. Sharp Liouville Theorems. Arxive Preprint, 2020.

[68] L. Wang, J. Wei, and S. Yan. On Lin-Ni's conjecture in convex domains. Proceedings of the London Mathematical Society, 102(6):1099-1126, 2011. 
[69] J. Wei and H. Chan. On De Giorgi's Conjecture: recent progress and open problems. Science China Mathematics, 61(11):1925-1946, 2018.

[70] E. Yanagida. Stability of stationary distributions in a space-dependent population growth process. Journal of Mathematical Biology, 15(1):37-50, sep 1982.

[71] E. Yanagida. Mini-maximizers for reaction-diffusion systems with SkewGradient Structure. Journal of Differential Equations, 179(1):311-335, feb 2002. 